\title{
A Phase Semantics for Polarized Linear Logic and Second Order Conservativity
}

\author{
Masahiro Hamano $^{*} \quad$ Ryo Takemura ${ }^{\dagger}$
}

December 27, 2008

\begin{abstract}
This paper presents a polarized phase semantics, with respect to which the linear fragment of second order polarized linear logic of [Laurent 99] is complete. This is done by adding a topological structure to Girard's phase semantics [Girard 87]. The topological structure results naturally from the categorical construction developed in [Hamano-Scott 07]. The polarity shifting operator $\downarrow$ (resp. $\uparrow$ ) is interpreted as an interior (resp. closure) operator in such a manner that positive (resp. negative) formulas correspond to open (resp. closed) facts. By accommodating the exponentials of linear logic, our model is extended to the polarized fragment of the second order linear logic. Strong forms of completeness theorems are given to yield cut-eliminations for the both second order systems. As an application of our semantics, the first order conservativity of linear logic is studied over its polarized fragment of [Laurent 02]. Using a counter model construction, the extension of this conservativity is shown to fail into the second order, whose solution is posed as an open problem in [Laurent 02]. After this negative result, a second order conservativity theorem is proved for an eta expanded fragment of the second order linear logic, which fragment retains a focalized sequent property of [Andreoli 92].
\end{abstract}

\section{Contents}

1 Introduction

2 Linear fragment MALLP2 of second order polarized linear logic 4

3 Polarized phase semantics 5

3.1 Phase space for MALL . . . . . . . . . . . . . . 5

3.2 Polarized phase space for MALLP . . . . . . . . . . . . 6

*Math Bio Unit, Okinawa Institute of Science and Technology, 12-2 Suzaki, Uruma, Okinawa, 904-2234, JAPAN. Email: hamano@oist.jp

${ }^{\dagger}$ Department of Philosophy, Keio University, 2-15-45 Mita, Minato-ku, Tokyo 108-8345, JAPAN. Email: takemura@abelard.flet.keio.ac.jp 
3.3 Second order polarized phase model for MALLP2 and completeness 10

3.4 Enriched phase space for LL . . . . . . . . . . . . . . . . . 12

3.5 Enriched polarized phase space for $L_{\text {pol }} \ldots \ldots$. . . . . . . . . 13

3.6 Second order polarized phase model for $\mathrm{LL}_{\mathrm{pol}} 2$ and completeness 15

4 Second order conservativity 16

4.1 First order conservativity . . . . . . . . . . . . . . . . 17

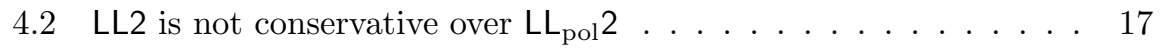

4.3 Second order $\eta$-expanded system LL ${ }^{\eta} 2 \ldots \ldots$. . . . . . . . . . . . . . 18

4.4 Main proposition for $\mathrm{LL}^{\eta} 2$ : Polarization . . . . . . . . . . . . 20

4.5 Additives . . . . . . . . . . . . . . . . . 26

4.6 Weakening and contraction ................ 26

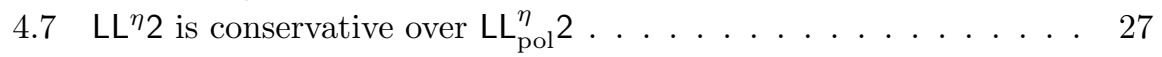

4.8 Some syntactical properties derived from Theorem 4.17 . . . . . 28

\section{Second order definability of restricted additives in polarized linear logic}

\section{Introduction}

Since Laurent's [1999] introduction of polarized linear logic, the notion of polarity of Girard [1991] has turned out to be an important hidden parameter controlling linear proof-theory. Laurent's formalization, in effect, provides a framework for explaining Andreoli's focusing proofs ([Andreoli 92]) in terms of the focalized sequent property. Danos-Joinet-Schellinx [1997] also relates polarity and focusing proofs for classical logic. The focalized sequent property of a logical system, which is not necessarily a polarized system, means that if a sequent is provable with only polarized formulas, especially in polarized linear logic, it contains at most one positive formula, in which case we call the sequent "focalized." Since the positive formula is always focused, each proof of polarized linear logic gives a focusing proof in Andreoli's sense. Laurent [2002] shows a first order conservativity theorem of linear logic LL over its polarized fragment $\mathrm{LL}_{\text {pol }}$. That is, if a (polarized) focalized sequent is provable in $\mathrm{LL}$, then it is also in $L L_{\text {pol }}$. Since all the proofs of $L L_{\text {pol }}$ are automatically focusing, it follows that any focalized sequent is provable with a focusing proof in LL. Combined with the focalized sequent property of $L L$, the conservativity neatly captures a main idea underlying polarity in linear logic: the "polarity" restriction on formulas leads naturally to focusing proofs. Moreover, seen from a logic programming viewpoint (cf. [Miller 04]), the conservativity is also important since we have only to work with focusing proofs. In his proof of the first order conservativity, Laurent made essential use of the subformula property of LL, which ensures that if a focalized sequent is provable then it is provable with only polarized formulas. When we try to extend the conservativity to the second order linear logic LL2, we immediately encounter a difficulty with the second order $\exists$-rule, which 
results in the loss of the subformula property. For this reason Laurent [2002] has left it open whether the conservativity result can be extended to second order.

In order to give an answer to the above question, we introduce a phase semantics for second order polarized linear logic. Phase semantics is introduced in [Girard 87] for an algebraic semantics of LL, and a noncommutative version is also studied in [Abrusci 91]. Phase semantics is weaker than either game or categorical semantics (e.g. [Laurent 02, Melliès 03, Hamano-Scott 07]) in the sense that only the notion of provability (and not that of proof) is considered. However, phase semantics has been quite useful to show various results for linear logic (eg. finite model property [Lafont 97], uniform treatment of cut-elimination [Okada 99, Terui 07], relationship to denotational model [Ehrhard 04]). In addition, compared with categorical semantics, phase semantics with its "simpler" structure is more naturally extendable to second order, as is seen in [Okada 99]. In particular, in the phase semantics of [Okada 99], a strong form of completeness theorem is given to yield the second order cut-elimination, for which syntactical methods hardly work. The main feature of our polarized phase semantics is its employment of a topological structure, which accommodates the two polarities as openness and closedness. This interpretation is an algebraic instance of the categorical construction developed in [Hamano-Scott 07] and is based upon the adjunction between interior and closure operators for the topology. A similar adjunction has recently been discussed in [Melliès 05] for game semantical studies of linear logic as well as in [Selinger 01] in his continuation-passingstyle models of $\lambda \mu$-calculus. Most recently after M. Hasegawa's observation, Melliès-Tabareau [2007] announce a categorization of phase semantics by using the continuation monad in their categorical semantics based on the adjunction. Few attempts have so far been made to clarify the notion of polarity in terms of topology, although as regards usual linear logic, topolinear spaces are studied for exponential connectives (e.g., [Girard 87, Sambin 95]). Indeed, to the best of our knowledge, no formulation of phase semantics for polarized linear logic has previously appeared in the literature. In the following, we introduce two kinds of second order topological phase semantics - a polarized phase semantics for multiplicative additive polarized linear logic MALLP2, and an enriched polarized phase semantics for $\mathrm{LL}_{\text {pol }} 2$ - and prove their strong completeness by using Okada's [1999] method, which implies second order cut-eliminations.

Then we first show by using a counter model construction that LL2 is not conservative over $\mathrm{LL}_{\mathrm{pol}} 2$ (Proposition 4.2), which is a rather unexpected byproduct of our polarized phase semantics. We next observe that LL2 does not have the focalized sequent property (Proposition 4.4). With these "negative" results, it appears that LL2 lacks the central idea of polarity in linear logic mentioned above, and that it offers no bridge between polarity and focusing. In order to remedy this shortcoming, we introduce an $\eta$-expanded fragment $\mathrm{LL}^{\eta} 2$ of LL2, in which atoms are exponential forms (i.e., ! $X^{\perp}$ (resp. ?X) for a positive (resp. negative) atom). Such a restriction, which was also adopted in [Laurent 99, 05a], has a natural semantical counterpart in our polarized phase spaces; a topological structure derived from the exponential connectives of LL coincides with a topological structure for the polarity. Moreover, syntactically, under the 
restriction, the focalized sequent property is recovered. Accordingly our main goal in this paper is to establish the conservativity of $\mathrm{LL}^{\eta} 2$ over its polarized fragment $\mathrm{LL}_{\mathrm{pol}}^{\eta} 2$ (Theorem 4.17). The conservativity follows from our main proposition (Proposition 4.16) which ensures that if a non polarized sequent is provable in $\mathrm{LL}^{\eta} 2$, then it is canonically decorated with! and ? so that the transformed polarized sequent is provable in $\mathrm{LL}_{\mathrm{pol}}^{\eta} 2$.

The rest of this paper is organized as follows. Section 2 provides a brief overview of MALLP2. In Section 3, we introduce two kinds of polarized phase semantics - one for MALLP and one for $\mathrm{LL}_{\mathrm{pol}}$ - and extend them to second order so as to yield complete models of MALLP2 and $\mathrm{LL}_{\mathrm{pol}} 2$, respectively. Section 4 is concerned with conservativity of first and second orders, and there the main theorem (Theorem 4.17) is established. In Section 5, we remark that restricted forms of the additives are definable in the second order multiplicative exponential polarised linear logic $\mathrm{MELL}_{\mathrm{pol}} 2$.

\section{Linear fragment MALLP2 of second order po- larized linear logic}

In this section, we review the second order multiplicative additive polarized linear logic MALLP2 of [Laurent 02]. In MALLP2, the exponentials ! and ? of the second order linear logic are replaced by the more primitive polarity shifting operators $\downarrow$ and $\uparrow$.

Formulas of MALLP2 are given by the following grammar:

$$
\begin{array}{ccc|c|c|c|c|c|c|c}
P & := & X & P \otimes P & P \oplus P & \mathbf{1} & \mathbf{0} & \downarrow N & \exists X . P \\
N & := & X^{\perp} & N \not D & N \& N & \perp & \top & \uparrow P & \forall X . N
\end{array}
$$

Notation: $P, Q$ (with or without subscript) (resp. $N, L$ ) denote positive (resp. negative) formulas; $A$ denotes any (positive or negative) formula. $\Gamma, \Delta$ denote multisets of formulas; $\mathcal{Q}($ resp. $\mathcal{N}$ ) denotes sequents consisting only of positive (resp. negative) formulas called positive sequents (resp. negative sequents).

Inference rules of MALLP2 are defined as follows:

$$
\begin{aligned}
& \frac{\vdash \Gamma, N \vdash N^{\perp}, \Delta}{\vdash N, N^{\perp}} \text { ax } c u t \\
& \frac{\vdash \Gamma, N, L}{\vdash \Gamma, N \mathcal{P} L} \not 8 \quad \frac{\vdash \Gamma, P \vdash \Delta, Q}{\vdash \Gamma, \Delta, P \otimes Q} \otimes \\
& \frac{\vdash \Gamma, N \quad \vdash \Gamma, L}{\vdash \Gamma, N \& L} \& \quad \frac{\vdash \Gamma, P}{\vdash \Gamma, P \oplus Q} \oplus 1 \quad \frac{\vdash \Gamma, Q}{\vdash \Gamma, P \oplus Q} \oplus 2 \\
& \overline{\vdash 1} 1 \quad \frac{\vdash \Gamma}{\vdash \Gamma, \perp} \perp \quad \overline{\vdash \Gamma, \top} \top \\
& \text { Here } \Gamma \text { contains at most } \\
& \text { one positive formula. }
\end{aligned}
$$




$$
\begin{aligned}
\frac{\vdash \mathcal{N}, N}{\vdash \mathcal{N}, \downarrow N} \downarrow & \frac{\vdash \Gamma, P}{\vdash \Gamma, \uparrow P} \uparrow \\
\frac{\vdash \Gamma, N}{\vdash \Gamma, \forall X . N} \forall & \frac{\vdash \Gamma, P[X:=Q]}{\vdash \Gamma, \exists X . P} \exists \\
\begin{array}{c}
X \text { does not appear free } \\
\text { in the lower sequent. }
\end{array} & Q \text { denotes a positive formula. }
\end{aligned}
$$

Note that in the $\exists$-rule the formula $Q$ substituted to $X$ is restricted to a positive formula.

Polarized linear logic has the following focalized sequent property, which is an important proof-theoretical property called the positive formula property in [Laurent 02].

Definition 2.1 (Focalized sequent and FSP) A sequent $\Gamma$ of polarized formulas is called focalized when it contains at most one positive formula.

A logical system $\mathcal{L}$, which is not necessarily a polarized system, has the focalized sequent property (FSP) if the following holds: if $\vdash \Gamma$ is provable in $\mathcal{L}$ with only polarized formulas and by restricting the $T$-rule to a focalized sequent, then $\Gamma$ is focalized.

Proposition 2.2 [Laurent 02, 99] MALLP2 has FSP.

\section{Polarized phase semantics}

This section introduces two kinds of topological phase spaces for first order logics; polarized phase spaces for MALLP in Section 3.2, and enriched polarized phase spaces for polarized linear logic $L_{\text {pol }}$ in Section 3.5. They are extended to second order so as to yield complete models of MALLP2 and $\mathrm{LL}_{\text {pol }} 2$, respectively.

\subsection{Phase space for MALL}

We first review the phase spaces of [Girard 87], with respect to which multiplicative additive linear logic MALL is complete.

Definition 3.1 (Phase space) A phase space is $M=(M, \perp)$ such that

- $M$ is a commutative monoid with $\cdot$ as its monoid operator and $\varepsilon$ as its unit element. The monoid operator - is lifted for subsets $\alpha, \beta \subseteq M$ as follows:

$$
\alpha \cdot \beta=\{x \cdot y \mid x \in \alpha, y \in \beta\} .
$$

$-\perp$ is a fixed subset of $M$. Then we define

$$
\alpha^{\perp}=\{x \mid x \cdot y \in \perp \text { for all } y \in \alpha\} .
$$

A fact is a subset $\alpha$ of $M$ such that $\alpha=\alpha^{\perp \perp}$. We denote the set of facts of $M$ by $D_{M \perp \perp}$ or simply by $D_{\perp \perp}$ when $M$ is clear from the context. 
For any $\alpha, \beta \subseteq M$, the following sets and operations are defined:

$$
\begin{aligned}
& \alpha \otimes \beta=(\alpha \cdot \beta)^{\perp \perp} \\
& \alpha \oplus \beta=(\alpha \cup \beta)^{\perp \perp} \\
& 1=\perp^{\perp}=\{\varepsilon\}^{\perp \perp} \\
& \mathbf{0}=\top^{\perp} \\
& \begin{array}{ll}
\alpha 8 \beta & =\left(\alpha^{\perp} \otimes \beta^{\perp}\right)^{\perp}=\left(\alpha^{\perp} \cdot \beta^{\perp}\right)^{\perp} \\
\alpha \& \beta & =\alpha \cap \beta \\
\alpha \multimap \beta & =\alpha^{\perp} 8 \beta=\left(\alpha \cdot \beta^{\perp}\right)^{\perp} \\
\top & =M
\end{array}
\end{aligned}
$$

See [Girard 87] for several properties which hold in phase spaces.

The set of facts $D_{\perp \perp}$ forms an algebraic example of $*$-autonomous category with products, whose objects are facts, and whose morphisms are set-inclusions among facts.

\subsection{Polarized phase space for MALLP}

In this subsection, we introduce a polarized phase semantics. Our motivation for the semantics is a simple question: What is a semantical counterpart of the syntactical notion of polarity in phase spaces? Our ingredient for the answer is a topological structure, which accommodates the notion of polarity so that positive and negative can be captured by the semantical notion of openness and closedness respectively in the topological structure. Thus the polarity shifting operator $\downarrow$ (dually $\uparrow$ ) is interpreted as an interior (resp. closure) operator. This interpretation stems from the categorical construction of [Hamano-Scott 07] when applied to phase spaces in particular. Let us start this subsection by defining our interior operator.

Definition 3.2 (Interior operator) Let $M=(M, \cdot, \varepsilon)$ be a commutative monoid. An interior $\downarrow$ is an operator from the power set $\mathcal{P}(M)$ of $M$ to $\mathcal{P}(M)$ which satisfies the following conditions. For any $\alpha, \beta \in \mathcal{P}(M)$,

1. (Intensivity) $\quad \downarrow \alpha \subseteq \alpha$

2. (Idempotency) $\downarrow \alpha \subseteq \downarrow \downarrow \alpha$

3. (Monotonicity) If $\alpha \subseteq \beta$, then $\downarrow \alpha \subseteq \downarrow \beta$

4. (Openness) $\quad \downarrow \alpha \cdot \downarrow \beta \subseteq \downarrow(\alpha \cdot \beta)$

Remark 3.3 (Interior operator) The usual definition of interior operator is (1), (2), and $\downarrow(\alpha \cap \beta)=\downarrow \alpha \cap \downarrow \beta$, which is stronger than our conditions (1), (2) and (3). Our condition (3) is enough to guarantee that an infinite union of open sets is open. See Proposition 3.14 below for a category-theoretical status of the operator.

Remark 3.4 (Openness of the monoid operator) A map $f: M \longrightarrow M$ is open if $f(\downarrow \alpha) \subseteq \downarrow(f(\alpha))$ holds for every $\alpha \in \mathcal{P}(M)$. Thus a binary map $f\left(x_{1}, x_{2}\right)$ is open if $f(\downarrow \alpha, \downarrow \beta) \subseteq \downarrow(f(\alpha, \beta))$ for every $\alpha, \beta \in \mathcal{P}(M)$. Hence the above condition (4) means that the monoid operator · is an open map.

A closure operator is defined, in a phase space, as the de Morgan dual operator of $\downarrow$. 
Definition 3.5 (Closure operator) Let $M=(M, \perp)$ be a phase space, and let $\downarrow$ be an interior operator. A closure $\uparrow: \mathcal{P}(M) \longrightarrow \mathcal{P}(M)$ is defined as an operator which satisfies the following conditions:

$$
\uparrow\left(\alpha^{\perp}\right)=(\downarrow \alpha)^{\perp} \quad \text { and } \quad(\uparrow \alpha)^{\perp}=\downarrow\left(\alpha^{\perp}\right) .
$$

We say that a set $\alpha$ is open (or $\downarrow$-invariant) if $\alpha=\downarrow \alpha$, and $\alpha$ is closed (or $\uparrow$-invariant) if $\alpha=\uparrow \alpha$.

Lemma 3.6 (Duality) For any fact $\alpha \in D_{\perp \perp}$,

$$
\uparrow \alpha=\left(\downarrow\left(\alpha^{\perp}\right)\right)^{\perp} .
$$

Proof. $\left(\downarrow\left(\alpha^{\perp}\right)\right)^{\perp}=\uparrow \alpha^{\perp \perp}=\uparrow \alpha$.

¿From Definition 3.5 and Lemma 3.6 we have the following duality.

Lemma $3.7 \alpha$ is closed iff $\alpha^{\perp}$ is open, for any fact $\alpha \in D_{\perp \perp}$.

Since $\downarrow$ and $\uparrow$ are de Morgan dual with respect to $\perp$ to each other, both operators are shown to preserve $\perp \perp$-invariance.

Lemma 3.8 ( $\downarrow$ and $\uparrow$ preserve $\perp \perp$-invariance) For any fact $\alpha \in D_{\perp \perp}$,

$$
\downarrow \alpha=(\downarrow \alpha)^{\perp \perp} \quad \text { and } \quad \uparrow \alpha=(\uparrow \alpha)^{\perp \perp} .
$$

Proof. $(\downarrow \alpha)^{\perp \perp}=\left(\uparrow \alpha^{\perp}\right)^{\perp}=\downarrow \alpha^{\perp \perp}=\downarrow \alpha . \quad(\uparrow \alpha)^{\perp \perp}=\left(\downarrow \alpha^{\perp}\right)^{\perp}=\uparrow \alpha^{\perp \perp}=\uparrow \alpha$.

Remark 3.9 (Closure operator of intuitionistic phase spaces and $\uparrow$ ) In studies of intuitionistic phase spaces, several authors [Abrusci 90, Troelstra 92, Ono 93, Okada-Terui 99] introduce closure operators by generalizing the ( $)^{\perp \perp}$ operator of classical linear logic. Although the de Morgan duals of such closure operators satisfy (1), (2) and (3) of Definition 3.2, they do not satisfy (4).

A polarized phase space is a phase space augmented with an interior operator.

Definition 3.10 (Polarized phase space) A polarized phase space is $M=$ $(M, \perp, \downarrow)$ such that

- $(M, \perp)$ is a phase space;

$-\downarrow$ is an interior operator such that

5. $\mathbf{1} \subseteq \downarrow \mathbf{1}, \quad$ where $\mathbf{1}=\perp^{\perp}$.

A polarized phase space yields the domains $D_{M+}$ (or simply $D_{+}$) of the set of open facts of $M$, i.e., $\left\{\alpha \in D_{M \perp \perp} \mid \alpha=\downarrow \alpha\right\}$, and $D_{M-}$ (or $D_{-}$) of the set of closed facts i.e., $\left\{\alpha \in D_{M \perp \perp} \mid \alpha=\uparrow \alpha\right\}$, where positive formulas and negative formulas are interpreted, respectively. 


\section{Proposition 3.11 (Adjunctions)}

$$
\begin{aligned}
\left(\ln j_{+} \dashv \downarrow\right) \text { For any } \alpha \in D_{+} \text {and } \beta & \in D_{\perp \perp,} \\
& \quad \alpha \subseteq \beta \quad \text { iff } \quad \alpha \subseteq \downarrow \beta
\end{aligned}
$$

That is, $\downarrow: D_{\perp \perp} \longrightarrow D_{+}$is right adjoint to $\ln j_{+}: D_{+} \hookrightarrow D_{\perp \perp}$.

$\left(\uparrow \dashv \mathrm{Inj}_{-}\right)$For any $\beta \in D_{\perp \perp}$ and $\gamma \in D_{-}$,

$$
\beta \subseteq \gamma \quad \text { iff } \quad \uparrow \beta \subseteq \gamma
$$

That is, $\uparrow: D_{\perp \perp} \longrightarrow D_{-}$is left adjoint to $\operatorname{lnj}_{-}: D_{-} \hookrightarrow D_{\perp \perp}$.

Note that the counit of the first adjunction is given by Intensivity (1) of Definition 3.2. These adjunctions give rise to the following adjunction $\Uparrow \dashv \Downarrow$ between $D_{+}$and $D_{-}$, where $\Downarrow=\downarrow \circ \ln j_{-}, \quad$ and $\Uparrow=\uparrow \circ \ln j_{+}$:

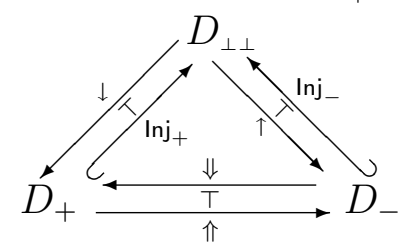

Alternatively, this diagram says that the full subcategory $\mathcal{D}_{-}$(resp. $\mathcal{D}_{+}$) of closed (resp. open) facts is a reflective (resp. coreflective) subcategory of the *-autonomous category of facts $\mathcal{D}_{\perp \perp}$ with a reflector $\uparrow$ (resp. coreflector $\downarrow$ ).

In polarized phase spaces, we have a nice semantical characterization for the polarity:

Proposition 3.12 (Positives are open; negatives are closed)

$\alpha \oplus \beta, \alpha \otimes \beta$ are both open for any open facts $\alpha, \beta$; and $\alpha \& \beta, \alpha \otimes \beta$ are both closed for any closed facts $\alpha, \beta$. Moreover, $\mathbf{1}=\perp^{\perp}$ and $\mathbf{0}=\top^{\perp}$ are open, and $\perp$ and $\top=M$ are closed. That is, the coreflective (resp. reflective) subcategory $\mathcal{D}_{+}$(resp. $\mathcal{D}_{-}$) of $\mathcal{D}_{\perp \perp}$ is closed under positive operations $\otimes, \oplus$, along with their respective units $\mathbf{1}$ and $\mathbf{0}$ (resp. under negative operations $88, \&$, along with their respective units $\perp$ and $\mathrm{T})$.

Proof. We prove only for $\otimes$ and $\oplus$. (Dually for $\not 8$ and $\&$, and the units are immediate.)

As for $\otimes$, by the openness of the monoid operator $\cdot$, we have $(\alpha \cdot \beta)^{\perp \perp}=$ $(\downarrow \alpha \cdot \downarrow \beta)^{\perp \perp} \subseteq(\downarrow(\alpha \cdot \beta))^{\perp \perp}=\downarrow\left((\alpha \cdot \beta)^{\perp \perp}\right)$. The other direction is immediate from the intensivity.

As for $\oplus$, using the fact $\alpha \subseteq(\alpha \cup \beta)^{\perp \perp}$ and the monotonicity, we have $\downarrow \alpha \subseteq$ $\downarrow\left((\alpha \cup \beta)^{\perp \perp}\right)$. Since the same applies to $\beta$, we have $\downarrow \alpha \cup \downarrow \beta \subseteq \downarrow\left((\alpha \cup \beta)^{\perp \perp}\right)$. Thus we have $(\alpha \cup \beta)^{\perp \perp}=(\downarrow \alpha \cup \downarrow \beta)^{\perp \perp} \subseteq\left(\downarrow\left((\alpha \cup \beta)^{\perp \perp}\right)\right)^{\perp \perp}=\downarrow\left((\alpha \cup \beta)^{\perp \perp}\right)$. The other direction is immediate from the intensivity.

Propositions 3.11 and 3.12 imply that the triple $\left(\mathcal{D}_{\perp \perp}, \mathcal{D}_{+}, \mathcal{D}_{-}\right)$forms an algebraic example of polarized $*$-autonomous category of [Hamano-Scott 07 ]. 
Remark 3.13 (Polarized $*$-autonomous category) A polarized *-autonomous category $\mathcal{C}_{+,-}$consists of a $*$-autonomous category with products $\mathcal{C}$, and a reflective (and a coreflective) full subcategory $\mathcal{C}_{-}\left(\right.$resp. $\left.\mathcal{C}_{+}\right)$of $\mathcal{C}$ with a reflector $\uparrow$ (resp. coreflector $\downarrow$ ). That is, there are distinguished functors $\uparrow: \mathcal{C} \longrightarrow \mathcal{C}_{-}$ and $\downarrow: \mathcal{C} \longrightarrow \mathcal{C}_{+}$satisfying: $\uparrow$ is left adjoint to the inclusion $\operatorname{Inj}_{-}: \mathcal{C}_{-} \hookrightarrow \mathcal{C}$, and $\downarrow$ is right adjoint to the inclusion $\operatorname{Inj}_{+}: \mathcal{C}_{+} \hookrightarrow \mathcal{C}$. (Cf. Proposition 3.11.) The subcategory $\mathcal{C}_{-}\left(\right.$and $\left.\mathcal{C}_{+}\right)$is required to be closed under negative (resp. positive) operations. (Cf. Proposition 3.12.) See [Hamano-Scott 07] for the detailed account.

Furthermore, category theory provides a nice characterization of our interior and closure operators on $D_{\perp \perp}$.

Proposition 3.14 (Interior as monoidal comonad) Let $\mathcal{D}_{\perp \perp}$ be the $*$-autonomous category of facts of a polarized phase space $M=(M, \perp, \downarrow)$. The interior (resp. closure) operator $\downarrow$ (resp. $\uparrow$ ) on $D_{\perp \perp}$ gives rise to a monoidal comonad (resp. comonoidal monad) over $\mathcal{D}_{\perp \perp}$, and vice versa.

Proof. We prove for $\downarrow$ (dually for $\uparrow$ ). By the equivalence of two concepts of adjunction and of comonad, the first adjunction $\left(\operatorname{Inj}_{+}, \downarrow, \eta, \epsilon\right)$ of Proposition 3.11 gives rise to a comonad $\downarrow=(\downarrow, \delta=\eta \downarrow, \epsilon)$ over $\mathcal{D}_{\perp \perp}$, and vice versa. The conditions (1) and (2) of Definition 3.2 correspond to the counit $\epsilon$ and the comultiplication $\delta$ of the comonad, respectively. The condition (3) describes the functoriality of the comonad. Moreover the conditions (4) and (5) correspond to the following functorial morphisms $\left(4^{\prime}\right)$ and (5') which describe monoidalness of the comonad:

$$
\begin{array}{ll}
4^{\prime} . & m_{\alpha, \beta}: \downarrow \alpha \otimes \downarrow \beta \stackrel{\eta_{\downarrow \alpha \otimes \downarrow \beta}}{\longrightarrow} \downarrow(\downarrow \alpha \otimes \downarrow \beta) \stackrel{\downarrow\left(\epsilon_{\alpha} \otimes \epsilon_{\beta}\right)}{\longrightarrow} \downarrow(\alpha \otimes \beta) \\
5^{\prime} . & m_{1}=\eta_{1}: \mathbf{1} \longrightarrow \downarrow \mathbf{1} .
\end{array}
$$

We give some examples of polarized phase spaces.

\section{Example 3.15 (Polarized phase space)}

1. For a submonoid $I$ of $M$, by defining

$$
\downarrow \alpha=(\alpha \cap I)^{\perp \perp},
$$

we have an interior operator. Note that this construction of an interior operator is the same as that of the exponential in an enriched phase space without weakening and contraction. (See Example 3.26 of Section 3.4.)

2. The multiplicative monoid $\mathbb{Z}_{3}=\mathbb{Z} / \mathbb{Z} 3$ of integers of modulo 3 yields a phase space $\left(\mathbb{Z}_{3},\{1,2\}\right)$ where $M=\mathbb{Z}_{3}$ and $\perp=\{1,2\}$. Moreover $\mathbb{Z}_{3}$ yields a polarized phase space: For a submonoid $I=\{1,2\}$, the interior operator $\downarrow$ is defined as $\downarrow \alpha=(\alpha \cap I)^{\perp \perp}$. In this polarized phase space, $\emptyset,\{1,2\}$ and $\{0,1,2\}$ are all facts, where $\emptyset$ is open, $\{1,2\}$ is clopen and $\{0,1,2\}$ is closed. 


\subsection{Second order polarized phase model for MALLP2 and completeness}

In this subsection, we extend our polarized phase spaces of Section 3.2 into second order so as to yield a complete semantics for MALLP2. We give a certain semantical restriction for the usual interpretation of the second order existential quantifier $\exists$ of [Okada 99]. Our restriction corresponds to the syntactical constraint for the $\exists$-rule in MALLP2, in which rule a formula substituted to the variable $X$ of $\exists X . P$ is restricted to be positive (rather than arbitrary as in the case of the second order linear logic LL2). We refer to [Okada 99] for the definition of second order phase semantics for LL2.

Notation: POS (resp. NEG) denotes the set of second order positive (resp. negative) formulas. $A\left[X_{1}, \ldots, X_{n}\right]$ (or more briefly $A[\vec{X}]$ ) means that the second order free variables of $A$ are contained in the list $X_{1}, \ldots, X_{n}$. We write $A[X:=$ $B$ ] for the substitution of $B$ for the free variables $X$ in $A$, and we abbreviate $A\left[X_{1}:=B_{1}, \ldots, X_{n}:=B_{n}\right]$ by $A[\vec{X}:=\vec{B}]$.

In order to interpret second order quantifiers, the following operations are introduced in the polarized phase spaces. Let $\bar{D}_{+} \subseteq D_{M+}$ and $\bar{D}_{-} \subseteq D_{M-}$. For any $\xi: \bar{D}_{+} \longrightarrow D_{M \perp \perp}$

$$
-\exists X . \xi(X)=\left(\bigcup_{\alpha \in \bar{D}_{+}} \xi(\alpha)\right)^{\perp \perp} \quad-\forall X . \xi(X)=\bigcap_{\alpha \in \bar{D}_{+}} \xi(\alpha)
$$

Proposition 3.12 for the first order connectives is extended to the second order.

Proposition 3.16 (Positives are open; negatives are closed)

$\exists X . \xi(X)$ is open for any $\xi: \bar{D}_{+} \rightarrow \bar{D}_{+}$, and $\forall X . \xi(X)$ is closed for any $\xi: \bar{D}_{+} \rightarrow \bar{D}_{-}$.

Proof. From $\xi(\alpha) \subseteq \bigcup_{\beta \in \bar{D}_{+}} \xi(\beta)$ for any $\alpha \in \bar{D}_{+}$, the adjunction of Proposition 3.11 applies to have $\xi(\alpha) \subseteq \downarrow \bigcup_{\beta \in \bar{D}_{+}} \xi(\beta)$ for any $\alpha \in \bar{D}_{+}$because $\xi(\alpha) \in$ $\bar{D}_{+}$. Thus we obtain $\bigcup_{\alpha \in \bar{D}_{+}} \xi(\alpha) \subseteq \downarrow \bigcup_{\beta \in \bar{D}_{+}} \xi(\beta)$, which means $\exists X . \xi(X) \subseteq$ $\downarrow \exists X . \xi(X)$. Dually for $\forall$.

Definition 3.17 (Second order phase model for MALLP2) A second order phase model $M=\left(M, \perp, \downarrow, \bar{D}_{+}, \bar{D}_{-}, *\right)$ for MALLP2 consists of

- a polarized phase space $(M, \perp, \downarrow)$;

- an interpretation function $*$ from the set of atoms of MALLP2 to the set $D_{M+}$ of open facts, which is extended to arbitrary formulas in a natural way;

- a subset $\bar{D}_{+}$of $D_{M+}$ such that $P^{*}[\vec{X}:=\vec{\alpha}] \in \bar{D}_{+}$for any $P \in P O S$ and for any $\alpha_{i} \in \bar{D}_{+}$;

- a subset $\bar{D}_{-}$of $D_{M-}$ such that $N^{*}[\vec{X}:=\vec{\alpha}] \in \bar{D}_{-}$for any $N \in N E G$ and for any $\alpha_{i} \in \bar{D}_{+}$. 
Note that, from Propositions 3.12 and 3.16, each positive (resp. negative) formula is interpreted by an open (resp. closed) fact.

The truth relation of sequents is defined by means of the set-theoretical inclusion relation between facts of the subdomain $\bar{D}_{+} \cup\{\perp\}$ of $D_{M \perp \perp}$. This semantical restriction on the subdomain naturally forces the truth relation of sequents to be defined only for the focalized sequents of Definition 2.1.

\section{Definition 3.18 (Truth value of sequents)}

A sequent $\Gamma[\vec{X}]$ is true, denoted $=\Gamma[\vec{X}]$ (or simply $=\Gamma$ when there is no need to indicate second order variables $\vec{X}$ ), if the following holds: For any $\beta_{i} \in \bar{D}_{+}$,

$$
\begin{array}{ll}
-N_{1}^{* \perp}[\vec{X}:=\vec{\beta}] \cdots N_{n}^{* \perp}[\vec{X}:=\vec{\beta}] \subseteq \perp & \text { when } \Gamma \text { is } N_{1}, \ldots, N_{n} \\
-N_{1}^{* \perp}[\vec{X}:=\vec{\beta}] \cdots \cdots N_{n}^{* \perp}[\vec{X}:=\vec{\beta}] \subseteq P^{*}[\vec{X}:=\vec{\beta}] & \text { when } \Gamma \text { is } N_{1}, \ldots, N_{n}, P
\end{array}
$$

Note that this interpretation of sequents is a restriction of that in phase semantics for MALL2 such that only focalized sequents are considered.

We have the soundness and the strong completeness theorems of MALLP2, which together imply the cut-elimination theorem in a way similar to that of [Okada 99].

Proposition 3.19 (Soundness of MALLP2) If $\vdash \Gamma$ is provable in MALLP2, then $\models \Gamma$ in any second order phase model for MALLP2.

Proof. By induction on the length of the proof of $\vdash \Gamma$ : The $\downarrow$-rule is obtained from the first adjunction of Proposition 3.11. The $\uparrow$-rule is obtained from the de Morgan dual of Intensivity (1) of $\downarrow$, which is extensivity of $\uparrow$. The other rules are treated in a way similar to that of [Okada 99].

In order to show the strong completeness theorem, we construct a syntactical model $M_{S}=\left(M_{S}, \perp, \downarrow, \bar{D}_{+}, \bar{D}_{-}, *\right)$ called a canonical model.

- $M_{S}$ is the monoid of the sequents of MALLP2 formulas, which are not restricted to focalized. Then $M_{S}$ is a monoid, whose monoid operator is the concatenation operator between sequents, and the unit is the empty sequent.

- We define the outer-value $\llbracket A \rrbracket$ for each formula $A$ as

$$
\llbracket A \rrbracket=\{\Gamma \Vdash \Gamma, A \text { is cut-free provable }\} .
$$

$-\perp:=\llbracket \perp \rrbracket=\{\Gamma \Vdash \Gamma, \perp$ is cut-free provable $\}=\{\Gamma \vdash \Gamma$ is cut-free provable $\}$.

- Let $I$ be the submonoid of $M_{S}$ consisting only of negative sequents. Then we define an interior operator $\downarrow$ as in Example 3.15(1):

$$
\downarrow \alpha=(\alpha \cap I)^{\perp \perp} .
$$

$-X^{*}=\llbracket X \rrbracket$ for each atom $X$. 
- For each positive formula $P$, let $\langle P\rangle$ be the set of open facts $\alpha$ of $M_{S}$ such that $P^{\perp} \in \alpha \subseteq \llbracket P \rrbracket$; and for each negative formula $N$, let $\langle N\rangle$ be the set of closed facts $\alpha$ such that $N^{\perp} \in \alpha \subseteq \llbracket N \rrbracket$. Then we define the second order domains $\bar{D}_{+}$by $\bigcup_{P \in P O S}\langle P\rangle$, and $\bar{D}_{-}$by $\bigcup_{N \in N E G}\langle N\rangle$.

By distinguishing the inner-value $A^{*}$, which is the interpretation of $A$ in the canonical model, and the outer-value $\llbracket A \rrbracket=\{\Gamma \Vdash \Gamma, A$ is cut-free provable $\}$, Okada [1999] proved the strong completeness theorem of LL2, which implies the cut-elimination theorem. This method is applicable to MALLP2.

Lemma 3.20 (Main lemma for MALLP2)

In $M_{S}$, for any $A[\vec{Y}]$, for any $\vec{Q} \in P O S$ and for any $\vec{\beta} \in\langle\vec{Q}\rangle$, we have

$$
A^{\perp}[\vec{Y}:=\vec{Q}] \in A^{*}[\vec{Y}:=\vec{\beta}] \subseteq \llbracket A[\vec{Y}:=\vec{Q}] \rrbracket .
$$

Proof. By induction on the complexity of $A$ as in [Okada 99].

Using the main lemma for MALLP2, we have the following strong completeness theorem.

Proposition 3.21 (Strong completeness of MALLP2) If $\models \Gamma$ in any second order phase model for MALLP2, then $\vdash \Gamma$ is provable without the cut-rule in MALLP2.

Combining this strong completeness theorem and the soundness theorem, we obtain the cut-elimination theorem for MALLP2.

Corollary 3.22 (Cut-elimination for MALLP2) If $\vdash \Gamma$ is provable in MALLP2, then it is provable without the cut-rule.

\subsection{Enriched phase space for $L L$}

In this subsection we review first order enriched phase spaces, with respect to which LL is complete ([Lafont 97]). Enriched phase spaces are obtained by augmenting the exponential! to phase spaces for MALL of Section 3.1. A key ingredient is the algebraic instance of Seely's axiomatization of the exponential in categorical model ([Seely 89]). Similar axiomatizations are found in the literatures of algebraic semantics [Troelstra 92, Ono 93, Abrusci 90].

Definition 3.23 (Enriched phase space) An enriched phase space $M=(M, \perp, !)$ is a phase space $(M, \perp)$ together with an exponential operator ! from $D_{\perp \perp}$ to $D_{\perp \perp}$ which satisfies the following conditions: For any $\alpha, \beta \in D_{\perp \perp}$,

1. (Intensivity) $\quad ! \alpha \subseteq \alpha$

2. (Idempotency) $\quad ! \alpha \subseteq ! ! \alpha$

3. (Monotonicity) If $\alpha \subseteq \beta$, then $! \alpha \subseteq ! \beta$

6. (Seely axiom 1$) \quad ! \alpha \otimes ! \beta=!(\alpha \& \beta)$

7. (Seely axiom 2) $\mathbf{1}=! \top$, where $T=M$

The dual operator ? of ! is defined as ? $\alpha=\left(!\left(\alpha^{\perp}\right)\right)^{\perp}$. 
In terms of category theory, our enriched phase spaces are characterized as follows:

Proposition 3.24 (Enriched phase space as Seely category) Let $\mathcal{D}_{\perp \perp}$ be the *-autonomous category of facts of an enriched phase space $M=(M, \perp, !)$. The exponential operator! gives rise to a comonad over $\mathcal{D}_{\perp \perp}$ so that $\left(\mathcal{D}_{\perp \perp}\right.$, !) forms a Seely category, and vice versa. In $\left(\mathcal{D}_{\perp \perp}\right.$, !), for each $\alpha \in \mathcal{D}_{\perp \perp}$, ! $\alpha$ is endowed with a comonoid structure, and, moreover, the comonad! is monoidal.

Proof. By the condition (3), the exponential gives rise to a functor ! from $\mathcal{D}_{\perp \perp}$ to $\mathcal{D}_{\perp \perp}$. Then natural transformations $\epsilon: ! \longrightarrow I d_{\mathcal{D}_{\perp \perp}}$ and $\delta: ! \longrightarrow$ ! $\circ$ ! are given by (1) and (2), respectively. Thus we obtain a comonad $!=(!, \delta, \epsilon)$ over $\mathcal{D}_{\perp \perp}$. (6) and (7) correspond to the Seely's natural isomorphisms.

The following morphisms (8) and (9) are derivable for each $\alpha \in \mathcal{D}_{\perp \perp}$, which show that $\left(! \alpha, d_{\alpha}, e_{\alpha}\right)$ is a comonoid in $\mathcal{D}_{\perp \perp}$ :

8. (Contraction) $d_{\alpha}: ! \alpha \stackrel{!\langle i d, i d\rangle}{\longrightarrow} !(\alpha \& \alpha)=! \alpha \otimes ! \alpha$

9. (Weakening) $e_{\alpha}: ! \alpha \stackrel{! *_{\alpha}}{\longrightarrow} ! \top=\mathbf{1}$, where $*_{\alpha}: \alpha \longrightarrow \top$ is the unique map to the terminal object $T$ of the posetal $\mathcal{D}_{\perp \perp}$.

In addition, the following morphisms are derivable, which describe monoidalness of the comonad !: For any $\alpha, \beta \in \mathcal{D}_{\perp \perp}$,

$$
\begin{aligned}
& \text { 4'. } m_{\alpha, \beta}: ! \alpha \otimes ! \beta=!(\alpha \& \beta) \stackrel{\delta}{\rightarrow} ! !(\alpha \& \beta)=!(! \alpha \otimes ! \beta) \stackrel{!(\epsilon \otimes \epsilon)}{\longrightarrow} !(\alpha \otimes \beta) \\
& \text { 5. } \quad m_{1}: \mathbf{1}=! \top \stackrel{\delta}{\longrightarrow} ! ! \top=! \mathbf{1}
\end{aligned}
$$

The conditions (1), (2), (3), (4'), and (5) indicate that the exponential ! induces an interior operator on $D_{\perp \perp}$. However to avoid the confusion, we do not call ! an interior operator, because in this paper we do not consider any topological structure derived from ! except those for which ! coincides with $\downarrow$ (cf. Definition 4.8 in Section 4.3).

Remark 3.25 (Seely axioms) Under the conditions (1), (2), and (3) of Definition 3.23, the Seely axioms (6) and (7) are equivalent to (4'), (5), (8) and (9). (Cf. [Ono 93].)

Example 3.26 (Example of an enriched phase space) Let $J=\{x \in \mathbf{1}$ $x \cdot x=x\}$ a submonoid of the domain $M$. If we define $! \alpha=(\alpha \cap J)^{\perp \perp}$, then we have an enriched phase space. This definition of ! is due to [Lafont 97].

\subsection{Enriched polarized phase space for $L L_{p o l}$}

In this subsection, we first review first order polarized linear logic $L L_{\text {pol }}$. Then we introduce enriched polarized phase spaces, with respect to which $L_{\text {pol }}$ is 
complete.

Formulas of $\mathrm{LL}_{\text {pol }}$ of [Laurent 02] are obtained by imposing the following polarity restriction on formulas of $\mathrm{LL}$ :

$$
\begin{array}{clc|c|c|c|c|c}
P & := & X & P \otimes P & P \oplus P & \mathbf{1} & \mathbf{0} & ! N \\
N & := & X^{\perp} & N \& N & N \& N & \perp & \top & ? P
\end{array}
$$

Inference rules of $L L_{\text {pol }}$ is the polarized fragment of $L L$ where the $T$-rule is restricted to a focalized sequent.

We write $? \mathcal{Q}$ for a negative sequent obtained from a positive sequent $\mathcal{Q}$ by putting ? to each formula of $\mathcal{Q}$.

$$
\begin{aligned}
& \frac{\vdash \Gamma, N \vdash N^{\perp}, \Delta}{\vdash N, N^{\perp}} \text { ax } c u t \\
& \frac{\vdash \Gamma, N, L}{\vdash \Gamma, N^{2} L} \not 8 \quad \frac{\vdash \Gamma, P \vdash \Delta, Q}{\vdash \Gamma, \Delta, P \otimes Q} \otimes \\
& \frac{\vdash \Gamma, N \quad \vdash \Gamma, L}{\vdash \Gamma, N \& L} \& \quad \frac{\vdash \Gamma, P}{\vdash \Gamma, P \oplus Q} \oplus 1 \quad \frac{\vdash \Gamma, Q}{\vdash \Gamma, P \oplus Q} \oplus 2 \\
& \overline{\vdash 1} \quad \frac{\vdash \Gamma}{\vdash \Gamma, \perp} \perp \quad \frac{\underset{ }{\vdash \Gamma, \top} \top}{\text { Here } \Gamma \text { contains at most }} \\
& \text { one positive formula. } \\
& \frac{\vdash ? \mathcal{Q}, N}{\vdash ? \mathcal{Q}, ! N} ! \quad \frac{\vdash \Gamma, P}{\vdash \Gamma, ? P} ? \quad \frac{\vdash \Gamma}{\vdash \Gamma, ? P} ? w \quad \frac{\vdash \Gamma, ? P, ? P}{\vdash \Gamma, ? P} ? c
\end{aligned}
$$

We now introduce enriched polarized phase spaces, with respect to which $\mathrm{LL}_{\text {pol }}$ is complete.

Definition 3.27 (Enriched polarized phase space) An enriched polarized phase space is $M=(M, \perp, !, \downarrow)$ such that

- $(M, \perp, !)$ is an enriched phase space;

- $(M, \perp, \downarrow)$ is a polarized phase space;

$-! \alpha \subseteq \downarrow \alpha$ for any fact $\alpha \in D_{\perp \perp}$.

Note that the topological structure considered in $M$ is that derived from $\downarrow$ (not from !).

Remark 3.28 (Two modalities $\downarrow$ and !) In enriched polarized phase spaces, the interior operator $\downarrow$ does not necessarily coincide with !. If it does, $X^{*} \subseteq$ !? $X^{*}$ holds for a positive atom $X$, which, however, happens to be a valid interpretation of an unprovable sequent $\vdash X^{\perp}, !$ ? $X$ in $\mathrm{LL}_{\text {pol }}$. (Cf. Definition 3.17.) 
Example 3.29 (Enriched polarized phase space) Let $I$ and $J$ be the submonoids of Example 3.15(1) and Example 3.26, respectively, and let $\downarrow$ be as in Example 3.15(1). Then by defining

$$
! \alpha=(\alpha \cap J \cap I)^{\perp \perp},
$$

we have an enriched polarized phase space. This essentially corresponds to the decomposition of the exponentials of [Girard 01]:

$$
! \alpha=\downarrow \sharp \alpha \quad \text { and } \quad ? \alpha=\uparrow b \alpha .
$$

Note that $\sharp \alpha$ is defined as $\alpha \cap J$, and then ! $\alpha$ is defined as the interior of $\sharp \alpha$ by Example 3.15(1). I.e., ! $\alpha=\downarrow \sharp \alpha=(\alpha \cap J \cap I)^{\perp \perp} . J \cap I \subseteq I$ yields the condition $! \alpha \subseteq \downarrow \alpha$. Note that $\sharp \alpha$ is not necessarily a fact, hence its meaning is only given under the connective $\downarrow$. (Cf. [Laurent 05b].)

Proposition 3.12 for MALLP connectives can be extended to $L L_{\text {pol }}$ connectives: i.e., we have the following Proposition 3.30 for the positive connective ! and the negative connective?

Proposition 3.30 (Positives are open; negatives are closed)

$! \alpha$ is open (i.e., $\downarrow$-invariant) and $? \alpha$ is closed (i.e., $\uparrow$-invariant) for any fact $\alpha$.

Proof. We show $! \alpha$ is open, i.e., $! \alpha=\downarrow ! \alpha$. We have $! \alpha \subseteq ! ! \alpha \subseteq \downarrow ! \alpha$ by Idempotency of ! and the definition ! $\alpha \subseteq \downarrow \alpha$. The other direction is immediate from the intensivity of $\downarrow$. Dually for ?.

This proposition says that the topology derived from ! is coarser than that from $\downarrow$. I.e., the set of !-invariant facts is a subset of $\downarrow$-invariant facts in the phase space $(M, \perp)$.

\subsection{Second order polarized phase model for $L L_{p o l} 2$ and completeness}

In this subsection, we extend our enriched polarized phase spaces of Section 3.5 into second order so as to yield a complete semantics for the second order extension $\mathrm{LL}_{\mathrm{pol}} 2$ of $\mathrm{LL}_{\mathrm{pol}}$.

The syntax of $L L_{\text {pol }} 2$ of [Laurent 02$]$ is obtained by adding the following rules for negative formulas $\forall X . N$ and for positive formulas $\exists X$.P to $\mathrm{LL}_{\mathrm{pol}}$ :

$$
\begin{array}{cc}
\frac{\vdash \Gamma, N}{\vdash \Gamma, \forall X . N} \forall & \frac{\vdash \Gamma, P[X:=Q]}{\vdash \Gamma, \exists X . P} \exists \\
\begin{array}{c}
X \text { does not appear free } \\
\text { in the lower sequent. }
\end{array} & Q \text { denotes a positive formula. }
\end{array}
$$

Definition 3.31 (Second order phase model for $L_{\mathrm{pol}} 2$ ) A second order phase model $M=\left(M, \perp, !, \downarrow, \bar{D}_{+}, \bar{D}_{-}, *\right)$ for $\mathrm{LL}_{\text {pol }} 2$ consists of 
- an enriched polarized phase space $(M, \perp, !, \downarrow)$;

- an interpretation function $*$ from the set of atoms of $\mathrm{LL}_{\mathrm{pol}} 2$ to the set $D_{M+}$ of open facts ;

- the second order domains $\bar{D}_{+}$and $\bar{D}_{-}$which are defined in the same way as for MALLP2. (See Definition 3.17.)

We have the soundness and the strong completeness theorems of $\mathrm{LL}_{\mathrm{pol}} 2$, which together imply the cut-elimination theorem in a way similar to that of [Okada 99].

Proposition 3.32 (Soundness of $\mathrm{LL}_{\mathrm{pol}} 2$ ) If $\vdash \Gamma$ is provable in $\mathrm{LL}_{\mathrm{pol}} 2$, then $=\Gamma$ in any second order phase model for $\mathrm{LL}_{\mathrm{pol}} 2$.

In order to show the completeness theorem, we construct the canonical model $M_{S}=\left(M_{S}, \perp, !, \downarrow, \bar{D}_{+}, \bar{D}_{-}, *\right)$ by adding the following definition of ! to the canonical model for MALLP2 of Section 3.3.

- Let $J$ be the submonoid of $M_{S}$ which consists only of negative sequents of the form ?Q. Here we identify more than one ?Q's with one ?Q in $M_{S}$ and $J$; for instance we identify ?Q,?Q with ?Q. Then we define $! \alpha=(\alpha \cap J)^{\perp \perp}$ for $\alpha \subseteq M_{S}$.

Since ? $\mathcal{Q}$ is a negative sequent, we have $J \subseteq I$, which implies $! \alpha \subseteq \downarrow \alpha$.

The strong completeness theorem which implies the cut-elimination theorem is shown by using the same form of the lemma (Lemma 3.20) as for MALLP2.

Lemma 3.33 (Main lemma for $\mathrm{LL}_{\mathrm{pol}} 2$ )

In $M_{S}$, for any $A[\vec{Y}]$, for any $\vec{Q} \in P O S$ and for any $\vec{\beta} \in\langle\vec{Q}\rangle$, we have

$$
A^{\perp}[\vec{Y}:=\vec{Q}] \in A^{*}[\vec{Y}:=\vec{\beta}] \subseteq \llbracket A[\vec{Y}:=\vec{Q}] \rrbracket .
$$

Proposition 3.34 (Strong completeness of $\mathrm{LL}_{\mathrm{pol}} 2$ ) If $\models \Gamma$ in any second order phase model for $\mathrm{LL}_{\mathrm{pol}} 2$, then $\vdash \Gamma$ is provable without the cut-rule in $\mathrm{LL}_{\mathrm{pol}} 2$.

Corollary 3.35 (Cut-elimination for $\mathrm{LL}_{\mathrm{pol}} 2$ ) If $\vdash \Gamma$ is provable in $\mathrm{LL}_{\mathrm{pol}} 2$, then it is provable without the cut-rule.

Remark 3.36 Let us consider an operator !- ${ }^{-}$which satisfies (1), (2), (3) of Definition 3.23 and (4'), (5) of Proposition 3.24. Then we can consider a complete subsystem $\mathrm{LL}_{\mathrm{pol}}^{-} 2$ which is the fragment of $\mathrm{LL}_{\mathrm{pol}} 2$ without the weakening and contraction rules. (Cf. Remark 4.18 of Section 4.7.)

\section{Second order conservativity}

This section is concerned with conservativity of linear logic. We briefly show the first order conservativity of $L L$ over $L_{\text {pol }}$ with a summary of four kinds of phase spaces. Then we consider a second order conservativity. In Section 4.3 , we introduce a fragment $\mathrm{LL}^{\eta} 2$ of $\mathrm{LL} 2$, then we prove our main technical proposition (Proposition 4.16) to obtain the main theorem (Theorem 4.17) of this paper: $L L^{\eta} 2$ is conservative over its polarized fragment $L_{\text {pol }}^{\eta} 2$. 


\subsection{First order conservativity}

Let us start by summarizing the four kinds of phase spaces for first order logics introduced so far (Figure 1). In the following figure, on the top of the most primitive phase spaces, three kinds of phase spaces are obtained, where a vertical (resp. horizontal) line designates the augmentation of the interior $\downarrow$ (resp. the exponential !). A symbol $\subset$ designates a subsystem relation: I.e., $L_{\text {pol }}$ and MALL are subsystems of LL.

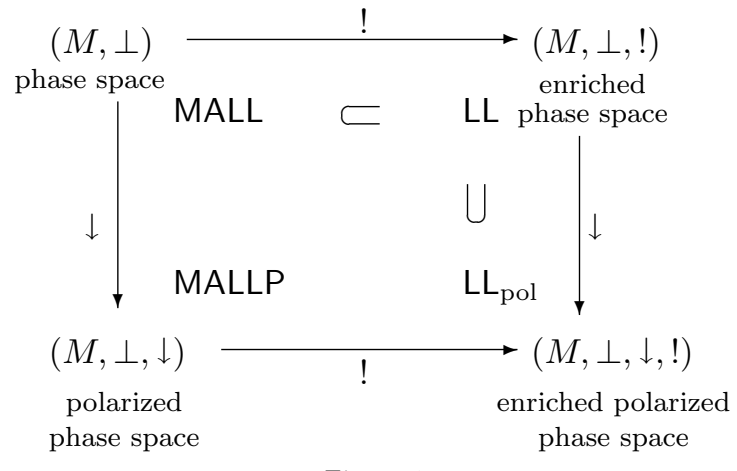

Figure 1

The conservativity theorem of first order $L L$ over $L_{\text {pol }}$ was first obtained by Laurent [2002 (p.50)] with a proof-theoretical argument. We have a simple semantical proof of the theorem as a corollary of the completeness theorem of $L L_{\text {pol }}$. The proof is direct by virtue of the canonical forgetting $\downarrow$ map from the bottom to the top on the right vertical relation of Figure 1.

Corollary 4.1 ( $\mathrm{LL} \succ \mathrm{LL}_{\text {pol }}$ [Laurent 02]) $\mathrm{LL}$ is conservative over $\mathrm{LL}_{\mathrm{pol}}$ : For any focalized sequent $\Gamma$, if $\vdash \Gamma$ is provable in $\mathrm{LL}$, then it is provable in $\mathrm{LL}_{\text {pol }}$.

Proof. Let $\Gamma$ be a focalized sequent which is provable in LL, and let $M=$ $(M, \perp, !, \downarrow)$ be an arbitrary enriched polarized phase space. Then from the soundness theorem of $\mathrm{LL}, \models \Gamma$ in the phase model $(M, \perp, !)$ for $\mathrm{LL}$. Since $\Gamma$ is polarized, it is also true in the polarized phase model $M$ for $\mathrm{LL}_{\text {pol }}$. Therefore, from the completeness theorem of $\mathrm{LL}_{\text {pol }}$, which is obtained as a corollary of the strong completeness and the cut-elimination theorems of $L L_{p o l} 2$, we have $\vdash \Gamma$ is provable in $\mathrm{LL}_{\text {pol }}$.

\subsection{LL2 is not conservative over $L_{\text {pol }} 2$}

In this subsection, we show that Corollary 4.1 does not extend to LL2. The semantic argument of Corollary 4.1 does not work for the second order LL2, because $=\exists X$.P in LL2 does not necessarily imply the same in $\mathrm{LL}_{\text {pol }} 2$. Note that $=\exists X$.P means $1 \subseteq P^{*}[X:=\alpha]$ for some $\alpha \in D_{\perp \perp}$ in $\mathrm{LL} 2$, but $\mathrm{LL}_{\mathrm{pol}} 2$ has the constraint that $\alpha$ ranges only over a subset $\bar{D}_{+}$of $D_{\perp \perp}$.

Proposition 4.2 (LL2 $\left.\nsucc \mathrm{LL}_{\mathrm{pol}} 2\right)$ LL2 is not conservative over $\mathrm{LL}_{\mathrm{pol}} 2$. 
Proof. We show that a focalized sequent $\vdash Y, ? \exists X . X$ is provable in LL2, but not in $\mathrm{LL}_{\mathrm{pol}}$ 2: The following is an LL2 poof of the sequent.

$$
\begin{aligned}
& \frac{{ }_{\vdash Y, Y^{\perp}} a x}{\vdash Y, \exists X . X} \exists\left(X:=Y^{\perp}\right) \\
& \frac{\vdash Y, ? \exists X . X}{\vdash}
\end{aligned}
$$

This LL2 proof is not an $L L_{\text {pol }} 2$ proof, since, in the application of the $\exists$-rule, the negative formula $Y^{\perp}$ is substituted to $X$. Note that this $\exists$-rule makes the LL2 provable sequent non focalized $(\vdash Y, \exists X . X)$.

In order to show the sequent is not provable in $\mathrm{LL}_{\text {pol }} 2$, we construct a counter model for $\vdash Y, ? \exists X . X$. Let $\mathbb{Z}_{3}$ be the enriched polarized phase space of Example $3.15(2)$ where $!=\downarrow$; and the second order domain $\bar{D}_{+}$is the set of open facts $\{\emptyset,\{1,2\}\}$. Let $Y^{*}$ be the open fact $\emptyset$. Then $Y^{\perp *}$ is the closed fact $\{0,1,2\}$. On the other hand, because $\exists X . X^{*}=\left(\bigcup_{\alpha \in \bar{D}_{+}} \alpha\right)^{\perp \perp}$, we have $? \exists X . X^{*}=\{1,2\}$. Hence, we have $\{0,1,2\}=Y^{\perp *} \nsubseteq \nsubseteq ? \exists X . X^{*}=\{1,2\}$ in this model. Therefore, we have $\not \models Y, ? \exists X . X$, and hence from the soundness theorem, we conclude that $\vdash Y, ? \exists X . X$ is not provable in $\mathrm{LL}_{\mathrm{pol}} 2$.

Remark 4.3 A direct syntactical proof of the unprovability of the sequent of Proposition 4.2 is also possible with the help of the second order cut-elimination theorem of $\mathrm{LL}_{\text {pol }} 2$ (Corollary 3.35), which follows from our strong completeness theorem.

As seen in the LL2 proof of $\vdash Y, ? \exists X . X$ of Proposition 4.2, a non focalized sequent $\vdash Y, \exists X . X$ is provable in LL2 with only polarized formulas. Thus we also do not have the focalized sequent property (FSP) in LL2.

Proposition 4.4 The focalized sequent property fails in LL2.

\subsection{Second order $\eta$-expanded system LL ${ }^{\eta} 2$}

In order to remedy the shortcoming of LL2 in the previous section, we introduce a fragment $\mathrm{LL}^{\eta} 2$, where atoms are restricted to exponential forms (i.e., of the form $! X^{\perp}$ (resp. ?X) for a positive (resp. negative) atom). Such a restriction of atoms is also adopted in [Laurent 99, 05a] for the first order $\mathrm{LL}_{\text {pol }}$.

Definition 4.5 ( $\left.L L^{\eta} 2\right)$ The syntax of $L L^{\eta} 2$ is defined as follows.

Formulas of $\mathrm{LL}^{\eta} 2$ are given by the following grammar.

$$
\begin{array}{cl|c|c|c|c|c|c}
A \quad:= & ! X^{\perp} & A \otimes A & A \oplus A & \mathbf{1} & \mathbf{0} & ! A & \exists X . A \\
& ? X & A \not 8 A & A \& A & \perp & \top & ? A & \forall X . A
\end{array}
$$

We refer to connectives $\{\otimes, \oplus, !, \exists, \mathbf{1}, \mathbf{0}\}$ (resp. $\{\not \mathcal{P}, \&, ?, \forall, \perp, \top\})$ as positive (resp. negative) connectives. 
The substitution of $B$ for the free occurrences of $X$ in $A$ (denoted $A[X:=B]$ ) is defined as usual for any $\mathrm{LL}^{\eta} 2$ formulas $A$ and $B$. Note that a substitution such as $X\left[X:=! Y^{\perp}\right]$ is not allowed in $\mathrm{LL}^{\eta} 2$ because $X$ is not an $\mathrm{LL}^{\eta} 2$ formula.

Inference rules of $L^{\eta}{ }^{\eta} 2$ are obtained from those of LL2 by replacing the usual axiom of the form $\vdash X, X^{\perp}$ with the form $\vdash ? X, ! X^{\perp}$.

The restriction will be crucial in proving Lemma 4.11 of the next subsection.

Example 4.6 (A proof in $\mathrm{LL}^{\eta} 2$ ) The following is an $\mathrm{LL}^{\eta} 2$ proof.

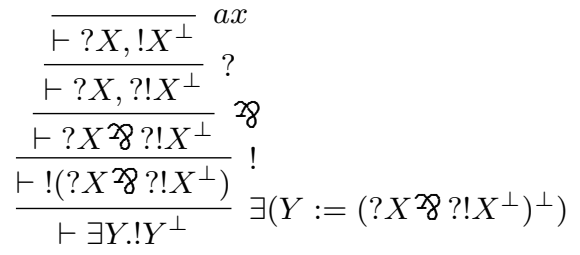

Since an application of the $\exists$-rule as in the proof of Proposition 4.2 is not allowed in $\mathrm{LL}^{\eta} 2$, the focalized sequent property (FSP) for $\mathrm{LL}^{\eta} 2$ follows naturally.

Lemma 4.7 LL ${ }^{\eta} 2$ has the focalized sequent property (FSP).

Proof. We prove this by induction on the given proof of $\mathrm{LL}^{\eta} 2$. Other than $\exists$-rule, the assertion is straightforward by the induction hypothesis. Let us consider a sequent $\vdash \exists X . P, \Gamma$ which is derived from a focalized sequent $\vdash P[X:=A], \Gamma$ via $\exists$-rule. We show that the formula $P[X:=A]$ is positive. This is because (i) if $P$ is atomic, it is of the form $! X^{\perp}$ in $\operatorname{LL}^{\eta} 2$, hence $! X^{\perp}[X:=A]$ is positive; (ii) otherwise it is clear because the sequent $\vdash P[X:=A], \Gamma$ is focalized. Thus we conclude that $\Gamma$ is negative, and hence the $\exists$-rule preserves FSP.

$\mathrm{LL}_{\text {pol }}^{\eta} 2$ denotes the polarized fragment of $\mathrm{LL}^{\eta} 2$. Then a phase model for $\mathrm{LL}_{\mathrm{pol}}^{\eta} 2$ is defined as follows.

Definition 4.8 (Second order phase model for $\mathrm{LL}_{\mathrm{pol}}^{\eta}$ ) A second order phase model $M=\left(M, \perp, !, \downarrow, \bar{D}_{+}, \bar{D}_{-}, *\right)$ for $\mathrm{LL}_{\mathrm{pol}}^{\eta} 2$ is a second order phase model for $\mathrm{LL}_{\mathrm{pol}} 2$ where

$-\downarrow$ coincides with !;

- an interpretation function is defined from the set of $\mathrm{LL}_{\mathrm{pol}}^{\eta}{ }^{2 \text {-atoms of the }}$ form ?X to the set of negative facts of the form ? $\alpha$ for $\alpha \in D_{\perp \perp}$.

Since two modalities $\downarrow$ and ! coincide, $\mathrm{LL}_{\text {pol }}^{\eta} 2$ is natural from our topological semantic viewpoint.

We have completeness of $\mathrm{LL}_{\text {pol }}^{\eta} 2$ in a way similar to that of $\mathrm{LL}_{\mathrm{pol}} 2$.

Proposition 4.9 (Strong completeness of $\left.\mathrm{LL}_{\mathrm{pol}}^{\eta} 2\right)$ If $\models \Gamma$ in any phase model for $\mathrm{LL}_{\text {pol }}^{\eta} 2$, then $\vdash \Gamma$ is provable without the cut-rule in $\mathrm{LL}_{\mathrm{pol}}^{\eta} 2$. 
Remark $4.10\left(\mathrm{LL}_{\mathrm{pol}}^{\eta} 2\right.$ is equivalent to $\left.\mathrm{LLP}{ }^{\eta} 2\right)$ LLP2 of [Laurent 02] is obtained by adding the following rules to $\mathrm{LL}_{\mathrm{pol}} 2$ :

$$
\frac{\vdash \mathcal{N}, N}{\vdash \mathcal{N}, ! N} N ! \quad \frac{\vdash \Gamma}{\vdash \Gamma, N} N w \quad \frac{\vdash \Gamma, N, N}{\vdash \Gamma, N} N c .
$$

LLP2 is not a subsystem of LL2 because the above $N !-, N w$ - and $N c$-rules strictly generalize the !-, ?w- and ?c-rules of $\mathrm{LL}_{\mathrm{pol}} 2$ respectively. These rules of $\mathrm{LL}_{\mathrm{pol}} 2$ are restrictions of the above LLP2 rules in the sense that negative formulas explicitly designated in the conclusions are of particular forms ?P. However when we consider $\eta$-expanded systems $\operatorname{LLP}^{\eta} 2$ and $\mathrm{LL}_{\mathrm{pol}}^{\eta} 2$, the above rules are shown to be derivable in $\mathrm{LL}_{\text {pol }}^{\eta} 2$, and hence $\mathrm{LLP}^{\eta} 2$ is equivalent to $\mathrm{LL}_{\mathrm{pol}}^{\eta} 2$.

\subsection{Main proposition for $L^{\eta}{ }^{\eta} 2$ : Polarization}

This subsection is devoted to proving Proposition 4.16, which is our main technical result in this paper. It is introduced to overcome the difficulty, remarked in [Laurent 02] (p. 51), with the following form of $\exists$-rule of $L^{\eta}{ }^{\eta} 2$ :

$$
\frac{\vdash_{\left\llcorner\eta_{2}\right.} \mathcal{N}, P[X:=A]}{\vdash_{\left\llcorner\eta_{2}\right.} \mathcal{N}, \exists X . P} \exists(X:=A) .
$$

Although the lower sequent is focalized, the upper sequent is no longer a polarized sequent because the formula $A$ is arbitrary. Our Proposition 4.16 ensures that the arbitrary $A$ is appropriately decorated with ! and ? to a positive formula $Q$ so that $\vdash \mathcal{N}, P[X:=Q]$ is provable in $\mathrm{LL}_{\text {pol }}^{\eta} 2$.

Throughout the proof of Proposition 4.16, we use the proof net representation of the proofs for the sake of simplicity. Since we do not use any particular properties of proof net in the following proof, we only indicate the links for $\mathrm{LL}^{\eta} 2$ proof net (without units) here. The details can be found in [Laurent 99,02, Laurent-Tortora 04].
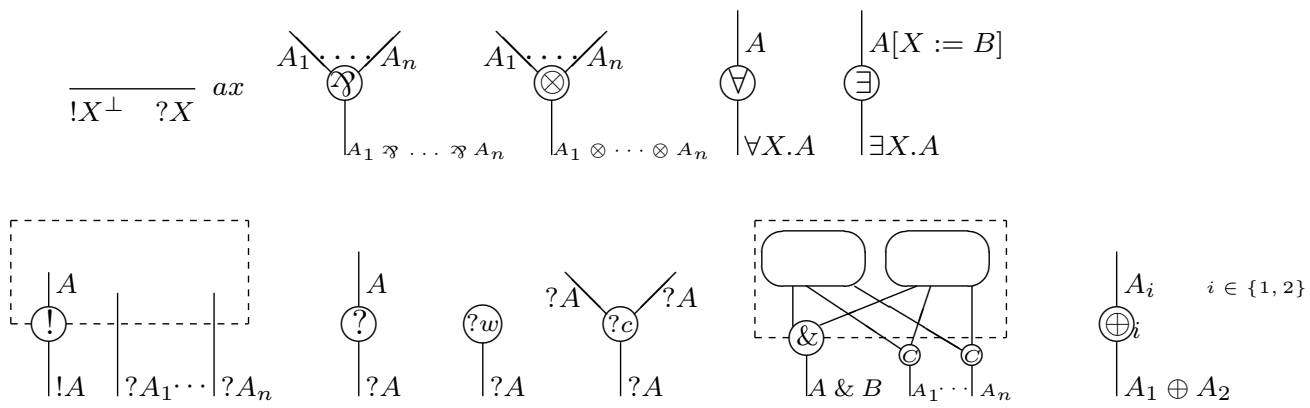

In the \&-box, ( $\odot$ 's are additive contraction nodes. We consider the 88 and $\otimes$ connectives as $n$-ary connectives for an appropriate $n$.

For the sake of simplicity of the argument, we do not consider any units $(\mathbf{1}, \mathbf{0}, \perp, \top)$ in the following proof.

We prepare some lemmas by recalling the following notions on proof nets. 
Splitting link: A terminal link $l$ of a proof net $\pi$ splits, if $l$ is removable to obtain proof nets which are premises of the corresponding rule to $l$. A formula $A$ in a conclusion of $\pi$ splits, if the corresponding terminal link of the outermost connective of $A$ splits. See [Andreoli-Maieli 99].

Andreoli's focusing property: Andreoli's focusing property says that a splitting conclusion $A$ can be chosen in such a way that each premises of $A$, if its outermost connective is positive, is again a splitting conclusion for the resulting proof nets after the splitting of $A$. I.e., whenever we write $A$ as $\phi\left[B_{1}, \ldots, B_{n}\right]$ by means of a cluster $\phi$ of positive connectives $\otimes, \oplus, \exists$, ! , then the corresponding links to the connectives successively split until reaching $B_{i}$ 's. Note that the crucial case is that outermost connectives of $B_{i}$ 's are no longer positive. See [Andreoli-Maieli 99, Andreoli 92, Laurent 05b]. $\mathrm{LL}^{\eta} 2$.

By virtue of Andreoli's focusing property, we have the following lemma in

Lemma 4.11 (Context lemma for a splitting ?-link) Let $A$ be an $\mathrm{LL}^{\eta} 2$ formula, and ? $\mathcal{Q}$ be an $\mathrm{LL}_{\text {pol }}^{\eta} 2$ sequent. For any $\mathrm{LL}^{\eta} 2$ proof net of conclusions $A, ? \mathcal{Q}$,

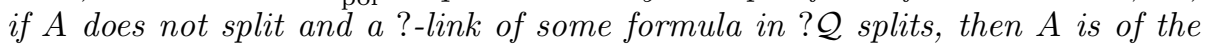
form ?B.

Proof. Assume $? \mathcal{Q}=? P, ? \mathcal{Q}_{1}$ so that, after the splitting of ?P, the positive $P$ splits. We consider the following cases according to the form of $P$ :

(i) If $P \equiv ! B_{1}$, the assertion is direct since the !-link of ! $B_{1}$ splits by the assumption. This means that $A$ lies on one of the auxiliary doors of the !-box as follows:

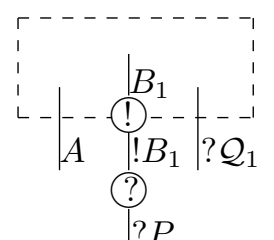

Hence $A$ is of the form ?B.

(ii) If $P \equiv B_{1} \otimes B_{2}, B_{1} \oplus B_{2}$ or $\exists X . B_{1}$, then $P$ splits to yield proof nets of conclusions $A, B_{i}[X:=C], ? \mathcal{Q}_{i}^{\prime}$ with $? \mathcal{Q}_{i}^{\prime} \subseteq$ ? $\mathcal{Q}_{1}$. We observe that the outermost connective of $B_{i}[X:=C]$ is either $\{\otimes, \oplus, \exists, !\}$; If $B_{i}[X]$ is non atomic, then the observation is clear because it is a subformula of $P$. If $B_{i}[X]$ is atomic, then it is of the form $! X^{\perp}$, hence the observation holds. From the observation, we consider the following two cases.

$(i i-a)$ If the outermost connective of $B_{i}[X:=C]$ is !, then, since the !-link splits from Andreoli's focusing property, the assertion holds from $(i)$.

$(i i-b)$ If the outermost connective of $B_{i}[X:=C]$ is $\otimes, \oplus$ or $\exists$, after successively applying this case $(i i)$ using Andreoli's focusing property, we finally obtain a proof net of conclusions $A, ! D, ? \mathcal{Q}_{i}^{\prime \prime}$ with $? \mathcal{Q}_{i}^{\prime \prime} \subseteq ? \mathcal{Q}_{1}$. Thus the assertion follows from $(i)$. 
Remark 4.12 Lemma 4.11 does not hold in (non $\eta$-expanded) LL2. The LL2 sequent $\vdash Y, ? \exists X . X$ in the proof of Proposition 4.2 gives a counterexample.

In what follows, we make the following syntactical convention.

Convention: $! P=P$ and $? N=N$ for any positive formula $P$ and for any negative formula $N$.

The convention is semantically valid in every polarized phase model of $\mathrm{LL}_{\text {pol }}^{\eta} 2$. This convention makes it possible to define uniformly the following canonical decoration $\sigma$.

Definition 4.13 (Canonical decoration) A canonical decoration $\sigma$ of $\mathrm{LL}^{\eta} 2$ formulas is defined inductively as follows, where $\sigma(A)$ is an $\mathrm{LL}_{\text {pol }}^{\eta} 2$ formula from the above convention.

$$
\begin{aligned}
& \sigma(? X)=? X \\
& \sigma(A \not 8 B)=? \sigma(A) \not 8 ? \sigma(B) \\
& \sigma(? A)=? ! \sigma(A) \\
& \sigma(\forall X . A)=\forall X . ? \sigma(A) \\
& \sigma\left(! X^{\perp}\right)=! X^{\perp} \\
& \sigma(A \otimes B)=! \sigma(A) \otimes ! \sigma(B) \\
& \sigma(! A)=! ? \sigma(A) \\
& \sigma(\exists X . A)=\exists X . ! \sigma(A) \\
& \sigma(A \& B)=? \sigma(A) \& ? \sigma(B) \\
& \sigma(A \oplus B)=! \sigma(A) \oplus ! \sigma(B)
\end{aligned}
$$

This decoration is the identity map on the class of polarized formulas in that $\sigma(A)=A$ for each polarized $\mathrm{LL}_{\mathrm{pol}}^{\eta} 2$ formula $A$.

We have the following lemma on the canonical decoration $\sigma$.

Lemma 4.14 (Substitution) Let $A$ and $B$ be $\mathrm{LL}^{\eta} 2$ formulas. In $\mathrm{LL}_{\mathrm{pol}}^{\eta} 2$, we have

$$
\sigma(A[X:=B]) \equiv \sigma(A)[X:=! \sigma(B)] .
$$

Note that, in the above lemma, the substituted formula $B$ is decorated by $\sigma$ into a positive formula $! \sigma(B)$, which serves to overcome the difficulty of the second order $\exists$-rule of $\mathrm{LL}^{\eta} 2$ mentioned in the beginning of this subsection.

Next we present the following lemma in preparation for the proof of Proposition 4.16. ${ }^{1}$

Lemma 4.15 For any $\mathrm{LL}^{\eta} 2$ formula $A$, and for any positive formulas $P$ and $Q$, the following sequents are provable in $\mathrm{LL}_{\mathrm{pol}}^{\eta} 2$ :

$$
\begin{aligned}
& \text { 1. !? } \sigma(A) \vdash ? ! \sigma(A) . \\
& \text { 2. ? } P[X:=Q] \vdash ? \exists X . P \text {. } \\
& \text { 3. ? } P_{i} \vdash ?\left(P_{1} \oplus P_{2}\right) \quad \text { for } i=1,2 .
\end{aligned}
$$

Proof. (1) If $\sigma(A)$ is positive, we have !? $\sigma(A) \vdash ? \sigma(A)=? ! \sigma(A)$. If $\sigma(A)$ is negative, we have !? $\sigma(A)=! \sigma(A) \vdash ? ! \sigma(A)$. (2) and (3) are straightforward.

Now we are ready to prove the main technical proposition of this paper:

${ }^{1}$ The lemma is used in the induction step for $n>1$ of Proposition 4.16: (1) for the case of ?B, (2) for the case of $\exists X . B$, and (3) for the case of $B_{1} \oplus B_{2}$. 
Proposition 4.16 (Polarization) Let $A_{1}, \ldots, A_{n}$ be $\mathrm{LL}^{\eta} 2$ formulas, and let $? \mathcal{Q}$ be an $\mathrm{LL}_{\text {pol }}^{\eta} 2$ sequent of the form $? Q_{1}, \ldots, ? Q_{m}$ with $m \geq 0$ (when $m=0$, ?Q is empty). If $\vdash A_{1}, \ldots, A_{n}, ? \mathcal{Q}$ is provable in $\mathrm{LL}^{\eta} 2$, then, in $\mathrm{LL}_{\text {pol }}^{\eta} 2$,

$$
\begin{array}{lll}
\vdash \sigma(A), ? \mathcal{Q} & \text { is provable } & \text { if } n=1 ; \text { and } \\
\vdash ? \sigma\left(A_{1}\right), \ldots, ? \sigma\left(A_{n}\right), ? \mathcal{Q} & \text { is provable } & \text { if } n>1 .
\end{array}
$$

Proof. In the following proof, $\vdash \Gamma$ means that there is an $\mathrm{LL}_{\mathrm{pol}}^{\eta}$ proof net of the conclusion $\Gamma$ (i.e., the sequent $\vdash \Gamma$ is provable in $\operatorname{LL}_{\text {pol }}^{\eta} 2$ ).

We prove the proposition by induction on the size of a cut-free proof net of conclusions $A_{1}, \ldots, A_{n}, ? \mathcal{Q}$. In order to make the proof easy to read, all the steps for the additive rules and for the weakening and the contraction rules are separately moved to the next sections (Section 4.5 and Section 4.6, respectively). As demonstrated there, to accommodate with these rules is straightforward. Before the proof, we recall that any terminal $\{\not 2 \mathcal{8}, \forall, \&\}$-link always splits to yield a proof net of smaller size.

Base step for $n=1$ :

This step is an axiom of the form ${\overline{! X^{\perp} ? X}}^{a x}$ so that $A \equiv ! X^{\perp}$ and $\mathcal{Q} \equiv X$. In this case the assertion is clear since $\sigma\left(! X^{\perp}\right)=! X^{\perp}$.

Base step for $n>1$ :

This step is the axiom of the form $\overline{! X^{\perp} ? X}$ ax so that $A_{1} \equiv ! X^{\perp}, A_{2} \equiv ? X$ and $? \mathcal{Q}$ is empty. We have $\vdash ? ! X^{\perp}, ? X$ where $? ! X^{\perp}=? \sigma\left(! X^{\perp}\right)$, and $? X=? \sigma(? X)$.

Induction step for $n=1$ :

We divide the following into two cases depending whether $A$ splits or not.

(Case 1) When $A$ splits, this case is divided according to the outermost connective of $A$.

If $A \equiv B_{1} \not 8 \ldots 88 B_{n}$, after removing the 18 -link, we have $\vdash ? \sigma\left(B_{1}\right), \cdots, ? \sigma\left(B_{n}\right), ? \mathcal{Q}$ by the induction hypothesis (I.H.). Then by the 28 -rules we have the assertion since $? \sigma\left(B_{1}\right)^{28} \ldots 88 ? \sigma\left(B_{n}\right)=\sigma\left(B_{1} 88 \ldots 88 B_{n}\right)$.

If $A \equiv \forall X$. B, after removing the $\forall$-link, we obtain $\vdash \sigma(B), ? \mathcal{Q}$ by I.H. Then we have $\vdash \forall X . ? \sigma(B), ? \mathcal{Q}$ by the ?-rule followed by the $\forall$-rule. This is the assertion since $\forall X . ? \sigma(B)=\sigma(\forall X . B)$.

If $A \equiv ? B$, each case is considered depending on the (kind of) splitting link of $? B$.

If the splitting link of ?B is the ?-link, after removing the ?-link, we have $\vdash \sigma(B), ? \mathcal{Q}$ by I.H. Then we have $\vdash ? ! \sigma(B), ? \mathcal{Q}$ by the !-rule followed by the ?-rule. Since ?! $\sigma(B)=\sigma(? B)$, we obtain $\vdash \sigma(? B), ? \mathcal{Q}$.

If $A \equiv ! B$, after removing the !-link, we have $\vdash \sigma(B), ? \mathcal{Q}$ by I.H. Then we obtain $\vdash ! ? \sigma(B), ? \mathcal{Q}$ by the ?-rule followed by the !-rule. Since !? $\sigma(B)=\sigma(! B)$, we obtain $\vdash \sigma(! B), ? \mathcal{Q}$.

If $A \equiv \exists X . B$, after removing the $\exists$-link, we obtain $\vdash \sigma(B[X:=C]), ? \mathcal{Q}$ by I.H. Using the substitution lemma (Lemma 4.14), we have $\vdash \sigma(B)[X:=$ $! \sigma(C)], ? \mathcal{Q}$, and hence we obtain $\vdash \exists X . ! \sigma(B), ? \mathcal{Q}$ by the !-rule followed by the $\exists$-rule. Since $\exists X . ! \sigma(B)=\sigma(\exists X . B)$, we obtain $\vdash \sigma(\exists X . B)$, ? $\mathcal{Q}$. 
If $A \equiv B_{1} \otimes \cdots \otimes B_{n}$, after removing the $\otimes$-link, we have $\vdash \sigma\left(B_{i}\right), ? \mathcal{Q}_{i}$ for all $1 \leq i \leq n$ by I.H. Then we have $\vdash ! \sigma\left(B_{i}\right), ? \mathcal{Q}_{i}$ by the !-rule, and hence we have $\vdash ! \sigma\left(B_{1}\right) \otimes \cdots \otimes ! \sigma\left(B_{n}\right), ? \mathcal{Q}$ by the $\otimes$-rules. Since $! \sigma\left(B_{1}\right) \otimes \cdots \otimes$ $! \sigma\left(B_{n}\right)=\sigma\left(B_{1} \otimes \cdots \otimes B_{n}\right)$, we obtain $\vdash \sigma\left(B_{1} \otimes \cdots \otimes B_{n}\right), ? \mathcal{Q}$.

(Case 2) When $A$ does not split, then ?P of ?Q $\mathcal{Q}$ splits. This case is divided depending on the (kind of) splitting link of ?P.

If the ?-link of ?P splits, ${ }^{2}$ note first that, in this case, by Lemma $4.11, A$ is of the form ?B, and the conclusions of the given $\mathrm{LL}^{\eta} 2$ proof net are written by $? B, ? P, ? \mathcal{Q}^{\prime}$. Then, after removing the ?-link, we have $\vdash ? \sigma(? B), ? \sigma(P), ? \mathcal{Q}^{\prime}$ by I.H. for the case $n>1$. Since $? \sigma(P)$ is ?P, and $? \sigma(? B)$ is $\sigma(? B)$, we obtain $\vdash \sigma(? B), ? P, ? \mathcal{Q}^{\prime}$.

Induction step for $n>1$ :

We divide the following into two main cases depending on whether $A_{1}, \ldots, A_{n}$ contains a splitting formula or not.

(Case 1) When there is no splitting formula among $A_{1}, \ldots, A_{n}$, then ?P in $? \mathcal{Q}$ splits. This case is divided depending on the (kind of) splitting link of ?P.

If the ?-link of ?P splits, after removing the ?-link, we have $\vdash ? \sigma\left(A_{1}\right), \ldots, ? \sigma\left(A_{n}\right)$, $? \sigma(P), ? \mathcal{Q}$ by I.H. Since $? \sigma(P)=? P$, we obtain $\vdash ? \sigma\left(A_{1}\right), \ldots, ? \sigma\left(A_{n}\right), ? P, ? \mathcal{Q}$.

(Case 2) When there is a splitting formula among $A_{1}, \ldots, A_{n}$, we further divide the following into two cases depending whether or not there is a terminal $\{\not \mathcal{B}, \forall, \&\}$-link in $A_{1}, \ldots, A_{n}$. In the following proof, $A$ denotes a certain $A_{i}$ by omitting its subscript, and $\Gamma$ denotes the sequence of other $A_{j}$ 's with $i \neq j$.

- When there is a terminal $\{\not \mathcal{P}, \forall, \&\}$-link in $A_{1}, \ldots, A_{n}$, note that such a negative terminal link always splits.

If $A \equiv B_{1}>8 \ldots 88 B_{n}$, then after removing the 88 -link, we have $\vdash ? \sigma\left(B_{1}\right), \ldots, ? \sigma\left(B_{n}\right), ? \sigma(\Gamma), ? \mathcal{Q}$ by I.H. Since $? \sigma\left(B_{1}\right) 28 \ldots 28 ? \sigma\left(B_{n}\right)=$ $? \sigma\left(B_{1}>8 \ldots 88 B_{n}\right)$, by the 28 -rules we obtain $\vdash ? \sigma\left(B_{1}>8 \ldots 8 B_{n}\right), ? \sigma(\Gamma), ? \mathcal{Q}$.

If $A \equiv \forall X$. B, then after removing the $\forall$-link, we have $\vdash ? \sigma(B), ? \sigma(\Gamma), ? \mathcal{Q}$ by I.H. Then by the $\forall$-rule, we have $\vdash \forall X . ? \sigma(B), ? \sigma(\Gamma), ? \mathcal{Q}$. Since $\forall X . ? \sigma(B)=$ $? \sigma(\forall X . B)$, we obtain $\vdash ? \sigma(\forall X . B), ? \sigma(\Gamma), ? \mathcal{Q}$.

- Otherwise we choose a splitting $A$ of $A_{1}, \ldots, A_{n}$ since such $A$ exists by the assumption. We consider each case according to the splitting link of $A$.

If $A \equiv ? B$ splits, each case is considered depending on the (kind of) splitting link of ?B.

If the ?-link of ?B splits, after removing the ?-link, we obtain $\vdash ? \sigma(B), ? \sigma(\Gamma), ? \mathcal{Q}$ by I.H. Then we have $\vdash ! ? \sigma(B), ? \sigma(\Gamma), ? \mathcal{Q}$ by the !-rule. By composing this with $! ? \sigma(B) \vdash ? ! \sigma(B)$ of Lemma $4.15(1)$ by the cut-rule, we have $\vdash ? ! \sigma(B), ? \sigma(\Gamma), ? \mathcal{Q}$. Thus $\vdash ? \sigma(? B), ? \sigma(\Gamma), ? \mathcal{Q}$ since $? ! \sigma(B)=? \sigma(? B)$.

\footnotetext{
${ }^{2}$ This case is the case where Lemma 4.11 is used.
} 
If $A \equiv ! B$ splits, after removing the !-link, we have $\vdash ? \sigma(B), ? \sigma(\Gamma), ? \mathcal{Q}$ by I.H.

Then we have $\vdash ? ! ? \sigma(B), ? \sigma(\Gamma), ? \mathcal{Q}$ by the !-rule followed by the ?-rule. Since ?!? $\sigma(B)=? \sigma(! B)$, we obtain $\vdash ? \sigma(! B), ? \sigma(\Gamma), ? \mathcal{Q}$.

If $A \equiv \exists X . B$ splits, after removing the $\exists$-link, we obtain $\vdash ? \sigma(B[X:=C]), ? \sigma(\Gamma), ? \mathcal{Q}$ by I.H. Then we further divide the following into two cases according to the polarity of $\sigma(B[X:=C])$ :

(i). If $\sigma(B[X:=C])$ is negative, we have $\vdash \sigma(B[X:=C]), ? \sigma(\Gamma), ? \mathcal{Q}$. Using the substitution lemma, we have $\vdash \sigma(B)[X:=! \sigma(C)], ? \sigma(\Gamma), ? \mathcal{Q}$, and hence $\vdash ! \sigma(B)[X:=! \sigma(C)], ? \sigma(\Gamma), ? \mathcal{Q}$ by the !-rule. Then, by the $\exists$-rule followed by the ?-rule, we have $\vdash ? \exists X . ! \sigma(B), ? \sigma(\Gamma), ? \mathcal{Q}$. Since $? \exists X . ! \sigma(B)=? \sigma(\exists X . B)$, we obtain $\vdash ? \sigma(\exists X . B), ? \sigma(\Gamma), ? \mathcal{Q}$.

(ii). If $\sigma(B[X:=C])$ is positive, using the substitution lemma, we have $\vdash ? \sigma(B)[X:=! \sigma(C)], ? \sigma(\Gamma), ? \mathcal{Q}$. By composing this with $? \sigma(B)[X:=$ $! \sigma(C)] \vdash ? \exists X . \sigma(B)$ of Lemma 4.15(2) by the cut-rule, we have $\vdash ? \exists X . \sigma(B), ? \sigma(\Gamma), ? \mathcal{Q}$. Since $? \exists X . \sigma(B)=? \sigma(\exists X . B)$, we obtain $\vdash ? \sigma(\exists X . B), ? \sigma(\Gamma), ? \mathcal{Q}$.

If $A \equiv B_{1} \otimes \cdots \otimes B_{n}$ splits, we divide the following into two cases according to the polarities of each $\sigma\left(B_{i}\right)$ for $1 \leq i \leq n$ :

(i). If all $\sigma\left(B_{1}\right), \ldots, \sigma\left(B_{n}\right)$ are negative, after removing the $\otimes$-link, we have $\vdash \sigma\left(B_{i}\right), ? \sigma\left(\Gamma_{i}\right), ? \mathcal{Q}_{i}$ for each $1 \leq i \leq n$ by I.H. Then by the !-rule we have $\vdash ! \sigma\left(B_{i}\right), ? \sigma\left(\Gamma_{i}\right), ? \mathcal{Q}_{i}$. By applying the $\otimes$-rules followed by the ?-rule, we have $\vdash ?\left(! \sigma\left(B_{1}\right) \otimes \cdots \otimes ! \sigma\left(B_{n}\right)\right), ? \sigma(\Gamma), ? \mathcal{Q}$. Since $?\left(! \sigma\left(B_{1}\right) \otimes \cdots \otimes ! \sigma\left(B_{n}\right)\right)=$ $? \sigma\left(B_{1} \otimes \cdots \otimes B_{n}\right)$, we obtain $\vdash ? \sigma\left(B_{1} \otimes \cdots \otimes B_{n}\right), ? \sigma(\Gamma), ? \mathcal{Q}$.

(ii). If some $\sigma\left(B_{i}\right)$ is positive, then $B_{i}$ is either $\exists X . C, ! C$ or $C_{1} \oplus C_{2}$. We show only the case of $\exists X . C$, and merely note that the other cases are similar.

If $B_{i}$ is $\exists X$.C, then from Andreoli's focusing property of $\mathrm{LL}^{\eta} 2$, this $\exists X . C$ splits to obtain the $\mathrm{LL}^{\eta} 2$ proof net of conclusions $C[X:=D], \Gamma_{i}, ? \mathcal{Q}_{i}$. Then by applying $\otimes$-rules to this proof net and to the other $(n-1)$-proof nets containing $B_{j}(j \neq i)$ having been obtained by the $\otimes$ splitting of this case, we obtain the following proof net in LL ${ }^{2} 2$ (i.e., the proof net is obtained from the original one by skipping the $\exists$-link):

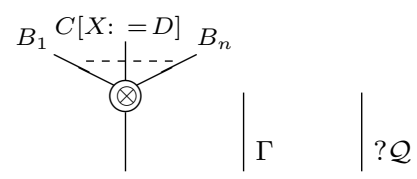

The size of this $\mathrm{LL}^{\eta} 2$ proof net is smaller than that of the original one, hence we have $\vdash ? \sigma\left(B_{1} \otimes \cdots \otimes C[X:=D] \otimes \cdots \otimes B_{n}\right), ? \sigma(\Gamma), ? \mathcal{Q}$ by I.H. On the other hand, using the substitution lemma, we have $! \sigma(C[X:=$ $D])=! \sigma(C)[X:=! \sigma(D)] \vdash \exists X . ! \sigma(C)$. Hence we have $\vdash ?\left(! \sigma\left(B_{1}\right) \otimes \cdots \otimes\right.$ $\left.\exists X . ! \sigma(C) \otimes \cdots \otimes ! \sigma\left(B_{n}\right)\right), ? \sigma(\Gamma), ? \mathcal{Q}$ by the cut-rule. Since $\exists X . ! \sigma(C)=$ $! \sigma(\exists X . C)$, we finally obtain $\vdash ? \sigma\left(B_{1} \otimes \cdots \otimes \exists X . C \otimes \cdots \otimes B_{n}\right), ? \sigma(\Gamma), ? \mathcal{Q}$. 


\subsection{Additives}

For the additive connectives $\&$ and $\oplus$, we add the following cases to the previous proof of Proposition 4.16.

\section{Induction step for $n=1$ :}

\section{(Case 1)}

If $A \equiv B_{1} \& B_{2}$, after removing the \&-link, we obtain $\vdash \sigma\left(B_{1}\right), ? \mathcal{Q}$ and $\vdash \sigma\left(B_{2}\right), ? \mathcal{Q}$ by I.H. Then by the ?-rule followed by the \&-rule, we have $\vdash ? \sigma\left(B_{1}\right) \& ? \sigma\left(B_{2}\right), ? \mathcal{Q}$. Thus we have the assertion since $\sigma \sigma\left(B_{1}\right) \& ? \sigma\left(B_{2}\right)=$ $\sigma\left(B_{1} \& B_{2}\right)$.

If $A \equiv B_{1} \oplus B_{2}$, after removing the $\oplus_{i}$-link, we obtain $\vdash \sigma\left(B_{i}\right), ? \mathcal{Q}$ for $i=1$ or $i=2$ by I.H. By the !-rule, we have $\vdash ! \sigma\left(B_{i}\right), ? \mathcal{Q}$, and hence we obtain $\vdash ! \sigma\left(B_{1}\right) \oplus ! \sigma\left(B_{2}\right), ? \mathcal{Q}$. Thus we have the assertion since $! \sigma\left(B_{1}\right) \oplus ! \sigma\left(B_{2}\right)=$ $\sigma\left(B_{1} \oplus B_{2}\right)$.

\section{Induction step for $n>1$ :}

\section{(Case 2)}

If $A \equiv B_{1} \& B_{2}$, then the \&-link is always splitting. Thus after removing the \&-link, we obtain $\vdash ? \sigma\left(B_{1}\right), ? \sigma(\Gamma), ? \mathcal{Q}$ and $\vdash ? \sigma\left(B_{2}\right), ? \sigma(\Gamma), ? \mathcal{Q}$ by I.H. Hence we obtain $\vdash ? \sigma\left(B_{1}\right) \& ? \sigma\left(B_{2}\right), ? \sigma(\Gamma), ? \mathcal{Q}$ by the \&-rule, which is the assertion since $? \sigma\left(B_{1}\right) \& ? \sigma\left(B_{2}\right)=? \sigma\left(B_{1} \& B_{2}\right)$.

If $A \equiv B_{1} \oplus B_{2}$ splits, after removing the $\oplus_{i}$-link for $i=1$ or 2 , we obtain $\vdash ? \sigma\left(B_{i}\right), ? \sigma(\Gamma), ? \mathcal{Q}$ by I.H. We assume $i=1$ without loss of generality. Then we further divide the following two cases according to the polarity of $\sigma\left(B_{1}\right)$.

$(i)$. If $\sigma\left(B_{1}\right)$ is negative, we have $\vdash \sigma\left(B_{1}\right), ? \sigma(\Gamma), ? \mathcal{Q}$. By the !-rule followed by the $\oplus_{1}$-rule, we have $\vdash ! \sigma\left(B_{1}\right) \oplus ! \sigma\left(B_{2}\right), ? \sigma(\Gamma), ? \mathcal{Q}$. Thus we obtain $\vdash ?\left(! \sigma\left(B_{1}\right) \oplus ! \sigma\left(B_{2}\right)\right), ? \sigma(\Gamma), ? \mathcal{Q}$, which is the assertion since $?\left(! \sigma\left(B_{1}\right) \oplus ! \sigma\left(B_{2}\right)\right)$ $=? \sigma\left(B_{1} \oplus B_{2}\right)$

(ii). If $\sigma\left(B_{1}\right)$ is positive, we obtain $\vdash ? \sigma\left(B_{1}\right), ? \sigma(\Gamma), ? \mathcal{Q}$. By composing this to $? \sigma\left(B_{1}\right) \vdash ?\left(\sigma\left(B_{1}\right) \oplus ! \sigma\left(B_{2}\right)\right)$ of Lemma $4.15(3)$ by the cut-rule, we obtain $\vdash ?\left(\sigma\left(B_{1}\right) \oplus ! \sigma\left(B_{2}\right)\right), ? \sigma(\Gamma), ? \mathcal{Q}$. Thus we have the assertion since $?\left(\sigma\left(B_{1}\right) \oplus ! \sigma\left(B_{2}\right)\right)=? \sigma\left(B_{1} \oplus B_{2}\right)$ in this case.

\subsection{Weakening and contraction}

In order to deal with the weakening and the contraction rules, we add the following cases to the previous proof of Proposition 4.16 in Section 4.4.

\section{Induction step for $n=1$ :}

\section{(Case 1)}

$A \equiv ? B$.

If the splitting link of ?B is the ?w-link, after removing the link, we obtain

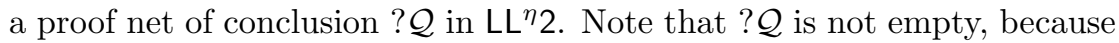


of the consistency of LL ${ }^{\eta} 2$ which is a consequence of the cut-elimination of $\mathrm{LL}^{\eta} 2$ (cf. [Okada 99]). Thus by I.H. we have $\vdash$ ?Q . Hence we have $\vdash ? ! \sigma(B), ? \mathcal{Q}$ where $? ! \sigma(B)=\sigma(? B)$.

If the splitting link of ?B is the ?c-link, after removing the link, we obtain $\vdash ? \sigma(? B), ? \sigma(? B), ? \mathcal{Q}$ by I.H. for the case of $n>1$. Thus we obtain $\vdash ? \sigma(? B), ? \mathcal{Q}$, and hence we have $\vdash \sigma(? B), ? \mathcal{Q}$ since $? \sigma(? B)=\sigma(? B)$.

(Case 2)

If the ?w-link of ?P splits, after removing the link, we obtain $\vdash \sigma(A), ? \mathcal{Q}$ by I.H. Thus we obtain $\vdash \sigma(A), ? P, ? \mathcal{Q}$ by the $? w$-rule.

If the ?c-link of ?P splits, after removing the link, we obtain $\vdash \sigma(A), ? P, ? P, ? \mathcal{Q}$ by I.H. Thus we obtain $\vdash \sigma(A), ? P, ? \mathcal{Q}$ by the ?c-rule.

\section{Induction step for $n>1$ :}

(Case 1)

If the ?w-link of ?P splits, we have the assertion by the same way as the induction step for $n=1$.

If the ?c-link of ? $P$ splits, we have the assertion by the same way as the induction step for $n=1$.

(Case 2)

$A \equiv ? B$.

If the ?w-link of ?B splits, after removing the link, we obtain $\vdash ? \sigma(\Gamma), ? \mathcal{Q}$ by I.H. Hence we obtain $\vdash ? ! \sigma(B), ? \sigma(\Gamma), ? \mathcal{Q}$ where $? ! \sigma(B)=? \sigma(? B)$.

If the ?c-link of ?B splits, after removing the link, we obtain $\vdash ? \sigma(? B), ? \sigma(? B), ? \sigma(\Gamma), ? \mathcal{Q}$ by I.H. Hence we obtain $\vdash ? \sigma(? B), ? \sigma(\Gamma), ? \mathcal{Q}$.

\section{7 $\mathrm{LL}^{\eta} 2$ is conservative over $\mathrm{LL}_{\mathrm{pol}}^{\eta} 2$}

Using Proposition 4.16, we show the main theorem of this paper.

Theorem $4.17\left(\mathrm{LL}^{\eta} 2 \succ \mathrm{LL}_{\mathrm{pol}}^{\eta} 2\right) \mathrm{LL}^{\eta} 2$ is conservative over $\mathrm{LL}_{\mathrm{pol}}^{\eta}$ 2. That is, for any focalized sequent $\Gamma$, if $\vdash \Gamma$ is provable in $\mathrm{LL}^{\eta} 2$, then it is provable in $\mathrm{LL}_{\text {pol }}^{\eta} 2$.

Proof. We prove the theorem by induction on the length of a given proof $\pi$ of $\vdash \Gamma$. If the last rule of $\pi$ is other than the $\exists$-rule, the assertion is straightforward from the induction hypothesis because the premises of the rules are focalized. So we consider the case where a focalized sequent $\vdash \exists X . P, \Gamma$ is provable from $\vdash P[X:=A], \Gamma$ via the $\exists$-rule. Since $\exists X . P$ is positive, $\Gamma$ is a negative sequent. Since negative connectives $\mathcal{R}, \forall$ and $\&$ are reversible, we may assume without loss of generality that $\Gamma$ is of the form ?QQ Then using Proposition 4.16 (when $n=1), \vdash \sigma(P[X:=A]), \Gamma$ is provable in $\mathrm{LL}_{\text {pol }}^{\eta}$. Since $\sigma(P[X:=A])=P[X:=$ $! \sigma(A)]$ by the substitution lemma (Lemma 4.14), $\vdash \exists X . P, \Gamma$ is provable by the $\exists$-rule of $\mathrm{LL}_{\mathrm{pol}}^{\eta} 2$. 
Remark 4.18 (Theorem 4.17 without weakening and contraction) A variation of Theorem 4.17 is also valid when the two systems are restricted to the fragments without weakening and contraction: I.e., let $\mathrm{LL}^{\eta-} 2$ be the fragment of $\mathrm{LL}^{\eta} 2$ without the weakening and the contraction rules. Then the variation states that $\mathrm{LL}^{\eta-} 2$ is conservative over its polarized fragment $\mathrm{LL}_{\text {pol }}^{\eta-} 2$. This variation is obtained because Proposition 4.16 holds for the fragment $L^{\eta-} 2$ by virtue of our proof method (see the first paragraph of the proof of the proposition).

\subsection{Some syntactical properties derived from Theorem 4.17}

In this subsection, as a consequence of Theorem 4.17, $\mathrm{LL}^{\eta} 2$ is shown to satisfy a stronger property than FSP (cf. Definition 2.1), which property we call the focalized proof property (FPP). This property explains a contrast between LL2 and LL ${ }^{\eta}$, which we have also seen in conservativities over their polarized fragments.

Definition 4.19 (Focalized proof property (FPP)) If a focalized sequent $\vdash \Gamma$ is provable in a logical system $\mathcal{L}$ by restricting $\top$-rules to focalized sequents, then there is a proof $\pi$ of $\vdash \Gamma$ which consists only of focalized sequents.

Remark 4.20 FPP is both FSP of Definition 2.1 and its reverse. The reverse property states as follows: If a focalized sequent $\vdash \Gamma$ is provable in $\mathcal{L}$ by restricting T-rules to focalized sequents, then there exists a proof $\pi$ of $\vdash \Gamma$ which consists only of polarized formulas.

Combining Theorem 4.17 and FSP of LL $\eta 2$ (cf. Lemma 4.7), we have:

Corollary 4.21 LL 2 has focalized proof property (FPP).

Proof. It suffices to show that $\mathrm{LL}^{\eta} 2$ has a property of Remark 4.20. Let $\vdash \Gamma$ be a focalized sequent which is provable in $\mathrm{LL}^{\eta} 2$. Then by the conservativity theorem (Theorem 4.17), $\vdash \Gamma$ is provable in $\mathrm{LL}_{\text {pol }}^{\eta}$, where proofs consist only of polarized formulas.

As shown in Proposition 4.4, LL2 does not have FSP, hence neither FPP. Let us summarize the states of these properties by the following table:

\begin{tabular}{|c|c|c|c|c|}
\hline & $\mathrm{LL}_{\mathrm{pol}}^{(\eta)} 2$ & LL & LL2 & $\mathrm{LL}^{\eta} 2$ \\
\hline FSP & Yes & Yes & $\begin{array}{c}\text { No } \\
\text { (Prop 4.4) }\end{array}$ & $\begin{array}{c}\text { Yes } \\
\text { (Lem 4.7) }\end{array}$ \\
\hline FPP & Yes & Yes & No & $\begin{array}{c}\text { Yes } \\
\text { (Cor 4.21) }\end{array}$ \\
\hline $\begin{array}{l}\text { Conservativity } \\
\text { over pol frag. }\end{array}$ & - & $\begin{array}{c}\text { Yes } \\
\text { (Cor 4.1) }\end{array}$ & $\begin{array}{c}\text { No } \\
\text { (Prop 4.2) }\end{array}$ & $\begin{array}{c}\text { Yes } \\
(\text { Thm 4.17) }\end{array}$ \\
\hline
\end{tabular}


Note that the left-most column of the table for $\mathrm{LL}_{\mathrm{pol}}^{(\eta)} 2$ is automatic. The column for LL is essentially due to [Laurent 02] and its FPP is obtained since the property of Remark 4.20 holds by virtue of the subformula property of the first order LL.

\section{Second order definability of restricted addi- tives in polarized linear logic}

In the polarized fragment $\mathrm{MELL}_{\text {pol }} 2$ of the second order multiplicative exponential linear logic, only restricted forms of additives are definable. Hence in Section 4 it is impossible to make additive connectives redundant.

By linearizing Prawitz's definition for the second order classical logic ([Prawitz $65]$ ), the additive connectives are known to be definable in MELL2 by the following and by its De Morgan dual:

$$
\begin{aligned}
& A \oplus B:=\forall X . !\left(A \multimap X^{\perp}\right) \multimap !\left(B \multimap X^{\perp}\right) \multimap X^{\perp} \\
& A \& B:=\exists X . !\left(A^{\not \mathcal{8}} X^{\perp}\right) \otimes !\left(B^{\not \mathcal{8}} X^{\perp}\right) \otimes X .
\end{aligned}
$$

By canonically polarizing these formulas, we define

$$
\begin{aligned}
P \hat{\oplus} Q & :=! \forall X . ?(P \otimes X)^{\not 8} ?(Q \otimes X)^{28} X^{\perp} \\
N \hat{\&} M & :=? \exists X . !\left(N^{\ngtr 8} X^{\perp}\right) \otimes !\left(M^{\ngtr 8} X^{\perp}\right) \otimes X .
\end{aligned}
$$

These then simulate the following restricted form of additive rules in $M E L L_{\text {pol }} 2$ :

$$
\frac{\vdash \Gamma, P_{i}}{\vdash \Gamma, P_{1} \hat{\oplus} P_{2}} \hat{\oplus}_{i=1,2} \quad \frac{\vdash \mathcal{N}, N \vdash \mathcal{N}, L}{\vdash \mathcal{N}, N \hat{\&} L} \hat{\&}
$$

I.e., in terms of categorical logic $\hat{\&}$ (resp. $\hat{\oplus}$ ) is a restriction of the usual (weaker) product (resp. coproduct) in $D_{+,-}$in the following sense: For $N, M \in \mathcal{D}_{-}$, $N \hat{\&} M$ is a triple $\left(N \hat{\&} M, N \hat{\&} M \stackrel{\text { proj }_{1}}{\longrightarrow} N, N \hat{\&} M \stackrel{\text { proj }_{2}}{\longrightarrow} M\right)$ such that the usual (weaker) universal mapping property for the product holds for an arbitrarily given triple $(P, P \longrightarrow N, P \longrightarrow M)$ for $P \in \mathcal{D}_{+}$. Note that the given triples are restrictions of the usual ones since $P$ ranges only in $\mathcal{D}_{+}$but not in $\mathcal{D}_{-}$.

It is impossible to extend the $\hat{\&}$-rule for a general context $\Gamma$ (not only $\mathcal{N}$ but also) containing at most one positive formula. This is because, the simulation of the \&-rule violates the focalized proof property of Definition 4.19, which MELL $L_{\text {pol }} 2$ retains (cf. Table 1 of Section 4.8).

The definability of the restricted additives $\hat{\oplus}$ and $\hat{\&}$ also works in $\mathrm{MELL}_{\text {pol }}^{\eta} 2$ if $X$ and $X^{\perp}$ are replaced by $! X^{\perp}$ and ?X, respectively. 


\section{Future work}

A promising future work is to give a truth-valued semantics for the indexed logical system MALLP $(I)$ of [Hamano-Takemura 08] by employing our polarized phase spaces. The syntactical system $\operatorname{MALLP}(I)$ is a polarized extension of [Bucciarelli-Ehrhard 00]'s indexed system and arises from multi-pointed relations, which form an web-based instance of the MALLP categorical model of [Hamano-Scott 07]. For this work an $I$-product polarized phase spaces are important analogously to [Ehrhard 04]. A product topology on the spaces is a key ingredient to accommodate the polarities to the indexed system.

As seen unexpectedly in Section 4, the second order syntaxes contrast with the first order ones in terms of conservativity and focalized sequent property. To understand this contrast, it is important to extend [Hamano-Scott 07]'s categorical semantics into second order MALLP2. A categorical model, which is stronger in modeling proofs, may explain this contrast observed in the weaker standpoint of provability, which conservativity as well as FSP concerns. Polarized dinatural transformation of [Hamano-Scott 07] is appropriate to model second order variable. It is also anticipated that incorporating second order in the categorical model provides a better understanding of focalization, whose semantical counter part is not yet clear.

The logical system MALLP2, which is complete with respect to our polarized phase spaces, is considered as the basic syntax of Girard's theory of ludics (without weakening). We intend to study a semantical strong normalization theorem for the L-nets of [Curien-Faggian 05] for ludics by using our polarized phase spaces.

\section{Acknowledgments}

The authors would like to thank Olivier Laurent, whose $\mathrm{PhD}$ thesis gave us many suggestions and who kindly read a preliminary version of the paper and gave us fruitful comments to improve the paper. The authors are grateful to anonymous referees for their helpful comments and criticisms, which make our presentations more comprehensive.

\section{References}

[Abramsky 03] S. Abramsky, Sequentiality vs. Concurrency In Games and Logic, Mathematical Structures in Computer Science, 13(4), 2003, 531-565.

[Abrusci 90] V. Michele Abrusci, Sequent calculus for intuitionistic linear propositional logic, Mathematical Logic, Plenum, New York, 1990, 223-242.

[Abrusci 91] V. Michele Abrusci, Phase Semantics and Sequent Calculus for Pure Noncommutative Classical Linear Propositional Logic, Journal of Symbolic Logic, Vol. 56, No. 4, 1991, 1403-1451. 
[Andreoli 92] Jean-Marc Andreoli, Logic Programming with Focusing Proofs in Linear Logic, Journal of Logic and Computation, 2(3), 297-347, 1992.

[Andreoli-Maieli 99] Jean-Marc Andreoli, Roberto Maieli, Focusing and ProofNets in Linear and Non-commutative Logic, Logic Programming and Automated Reasoning, 321-336, 1999.

[Asperti-Longo 92] Andrea Asperti and Giuseppe Longo, Categories, Types, and Structures: An Introduction to Category Theory for the Working Computer Scientist Foundations of Computing Series, M.I.T. Press, 1991.

[Bucciarelli-Ehrhard 00] Antonio Bucciarelli and Thomas Ehrhard, On phase semantics and denotational semantics in multiplicative-additive linear logic, Annals of Pure and Applied Logic, 102(3), 247-282, 2000.

[Curien-Faggian 05] Pierre-Louis Curien and Claudia Faggian, L-nets, strategies and proof-nets, Computer Science Logic 05, LNCS, Springer, 167-183, 2005 .

[Danos-Joinet-Schellinx 97] Vincent Danos, Jean-Baptiste Joinet, Harold Schellinx, A New Deconstructive Logic: Linear Logic, Journal of Symbolic Logic, 62(3), 755-807, 1997.

[Ehrhard 04] Thomas Ehrhard, A completeness theorem for symmetric product phase spaces, Journal of Symbolic Logic, Volume 69, Issue 2, 2004, 340-370.

[Girard 87] Jean-Yves Girard, Linear Logic, Theoretical Computer Science, 50, 1-102, 1987.

[Girard 91] Jean-Yves Girard, A New Constructive Logic: Classical Logic, Mathematical Structures in Computer Science, 1 (3), 255-296, 1991.

[Girard 01] Jean-Yves Girard, Locus Solum: From the rules of logic to the logic of rules, Mathematical Structures in Computer Science, 11(3), 301-506, 2001.

[Hamano-Scott 07] Masahiro Hamano and Philip Scott, A Categorical Semantics for Polarized MALL, Annals of Pure and Applied logic, 145, 2007, 276-313.

[Hamano-Takemura 08] Masahiro Hamano and Ryo Takemura, An Indexed System for Multiplicative Additive Polarized Linear Logic, Computer Science Logic 08, LNCS 5213, Springer Verlag, 262-277, 2008.

[Lafont 97] Yves Lafont, The Finite Model Property for Various Fragments of Linear Logic, Journal of Symbolic Logic, 62(4), 1202-1208, 1997.

[Laurent 99] Olivier Laurent, Polarized Proof-Nets: Proof-Nets for LC, TLCA $1999,213-227$.

[Laurent 02] Olivier Laurent, Étude de la polarisation en logique, Thèse de Doctorat, Institut de Mathématiques de Luminy, Université Aix-Marseille II, 2002 . 
[Laurent 05a] Olivier Laurent, Syntax vs. semantics: A polarized approach, Theoretical Computer Science, 343 (1-2), 177-206, 2005.

[Laurent 05b] Olivier Laurent, A proof of the focalization property of Linear Logic, draft, 2005.

[Laurent-Tortora 04] Olivier Laurent, Lorenzo Tortora de Falco, Slicing polarized additive normalization, in Linear Logic in Computer Science, eds. T. Ehrhard, J.-Y. Girard, P. Ruet, and P. Scott, LMS Lecture Series 316, Cambridge University Press, 2004, 247-282.

[Melliès 03] Paul-André Melliès, Categorical models of linear logic revisited, Prépublication de l'équipe PPS (September 2003, number 22), to appear in Theoretical Computer Science.

[Melliès 05] Paul-André Melliès, Asynchronous games 3, An innocent model of linear logic, in CTCS04, Electronic Notes in Theoretical Computer Science, Mar 2005.

[Melliès-Tabareau 07] Paul-André Melliès and Nicolas Tabareau, Resource modalities in game semantics, to appear in the proceedings of the 23th Conference on Logic in Computer Science (LICS 2007), Wroclaw, 2007.

[Miller 04] Dale Miller, An overview of linear logic programming, in Linear Logic and Computer Science, eds. T. Ehrhard, J.-Y. Girard, P. Ruet, and P. Scott, LMS Lecture Series 316, Cambridge University Press, 2004, 119-150.

[Okada 99] Mitsuhiro Okada, Phase Semantic Cut-Elimination and Normalization Proofs of First- and Higher-Order Linear Logic, Theoretical Computer Science, 227(1-2), 1999, 333-396.

[Okada-Terui 99] Mitsuhiro Okada and Kazushige Terui, The Finite Model Property for Various Fragments of Intuitionistic Linear Logic, Journal of Symbolic Logic, 64 (1999), 790-802.

[Ono 93] Hiroakira Ono, Semantics for substructural logics, in Substructural Logics, K. Došen and P. Schroeder-Heister ed., Oxford University Press, 1993, 259-291.

[Prawitz 65] Dag Prawitz, Natural Deduction - A proof theoretical study, Almquist and Wiksell, Stockholm, 1965.

[Sambin 95] Giovanni Sambin, Pretopologies and Completeness Proofs, Journal of Symbolic Logic, Vol. 60, No. 3, 1995, 861-878.

[Seely 89] R. A. G. Seely, Linear logic, *-autonomous categories and cofree coalgebras, in: J. Gray and A. Scedrov (eds.), Categories in Computer Science and Logic, Contemporary Mathematics 92, 1989, 371-382. 
[Selinger 01] Peter Selinger, Control categories and duality: on the categorical semantics of the lambda-mu calculus, Mathematical Structures in Computer Science, 11, 2001, 207-260.

[Terui 07] Kazushige Terui, Which Structural Rules Admit Cut Elimination? —An Algebraic Criterion, Journal of Symbolic Logic, 72 (3) 2007, 738-754.

[Troelstra 92] A. S. Troelstra, Lectures on Linear Logic, CSLI Lecture Notes, 1992, vol. 29, Stanford. 